\title{
Seabed foundation stability around offshore detached breakwaters
}

\author{
Lin Cui ${ }^{\mathrm{a}, *}$, Dong-Sheng Jeng ${ }^{\mathrm{b}}$, Junwei Liu ${ }^{\mathrm{a}}$ \\ ${ }^{a}$ College of Civil Engineering, Qingdao University of Technology, Qingdao, 266033, China \\ ${ }^{b}$ School of Engineering $\mathcal{E}$ Built Environment, Griffith University Gold Coast Campus, QLD 4222, Australia
}

5 Abstract

In this study, a three-dimensional (3D) model is adopted to investigate the problem of fluid-seabed-structure interactions (FSSI) around offshore detached breakwaters. The Volume-Averaged Reynolds-Averaged Navier-Stokes (VARANS) equations are used to simulate the 3D fluid motion in the vicinity of breakwaters, including reflected, diffracted waves and non-linear turbulence. The partially dynamic Biot's equations (i.e., the $u-p$ approximations) and poro-elastoplastic constitutive model are adopted to describe fluid-seabed interactions in a porous seabed. A one-way coupling algorithm is established to integrate the fluid motion and seabed response. By using the present model, this study addresses the long term cyclic loading induced seabed response and liquefaction potential of a loosely packed foundation. In addition, the effects of the perpendicular longshore currents on the hydrodynamic properties and liquefaction potential are also taken into account. The numerical results reveal that the loose sandy seabed around the offshore detached breakwaters experienced a gradual decrease of stiffness until liquefaction occurs under successive loading cycles. Longshore currents tend to exacerbate the wave field and increase the risk of liquefaction of seabed foundation, especially the shallow soil layer near the surface.

6 Keywords: Fluid-Seabed-Breakwater Interactions; VARANS equations; Poro-elastoplastic model; Liquefaction; 7 Loosely deposited seabed foundation

\section{Introduction}

Offshore detached breakwaters have been widely constructed as one of the coastal management facilities to

\footnotetext{
${ }^{*}$ Corresponding author.

Email addresses: lin.cui3@griffithuni . edu.au (Lin Cui), d.jeng@griffith. edu . au (Dong-Sheng Jeng), liujunwei@qut.edu.cn (Junwei Liu) 
To date, significant efforts have been devoted to the problem of FSSI around breakwaters using different methods, including analytical solutions with the boundary-layer approximation (Mynett and Mei, 1982; Tsai et al., 1990; Kumagai and Foda, 2002), numerical simulations (Jeng et al., 2000, 2001; Ulker et al., 2010, 2012; Ye et al., 2014; Zhao et al., 2018, 2020a,b) and physical modelling (Jeng et al., 2005; Kudella et al., 2006; Chang et al., 2007). However, most previous studies were limited to two-dimensional models whose object is often a typical trunk section of a long breakwater.

For case around breakwater heads, or in the vicinity of the segmented breakwaters, the three-dimensional (3D) FSSI phenomenon, involving more complicated flow regime, is significant different from the aforementioned twodimensional cases (Fredsøe and Sumer, 1997). Numerous researchers considered the 3D seabed responses around breakwaters since 1996. For example, Jeng (1996) analytically solved the wave-induced oscillatory soil response in the vicinity of breakwater heads in a poro-elastic seabed. Li and Jeng (2008) proposed a finite difference model for residual soil response in the vicinity of breakwater heads. Jeng and Ou (2010) developed a three-dimensional poro-elastoplastic finite element model to study the wave-induced pore pressure around breakwater heads. They compared the pore water pressure and liquefaction development in both elastic and elastoplastic seabeds and found that the elastic model will underestimate the size of liquefaction zone around breakwater heads. However, in these three studies (Jeng, 1996; Li and Jeng, 2008; Jeng and Ou, 2010), the 3D wave loading around breakwater heads was adopted as analytical solution (Stoker, 1957), in which the breakwater was treated as an infinite line. Later, Zhao et al. (2013) developed a 3D model to study the wave-induced pore pressures in a porous seabed around breakwater heads. Unlike the previous works, Zhao et al. (2013) used Navier-Stokes equations to solve the flow field in the vicinity of the breakwaters. Later, a few researchers tried to further investigated the three-dimensional seabed response around breakwaters under wave loading (Ye et al., 2016; Liao et al., 2018).

Two mechanisms of the wave-induced seabed responses have been identified through field observations and laboratory experiments, namely oscillatory mechanism and residual mechanism (Sumer, 2014; Jeng, 2018). Most available FSSI models tend to focus on the first mechanism, which mostly are based on the poro-elastic theory. In such models, the seabed is treated as a poro-elastic material that does not consider any compaction of the soil, only the oscillation of pore pressures, attenuation of pressure amplitude and phase lags can be observed. The residual mechanism plays a dominant role in the seabed with small relative density, low strength, poor drainage and easy to accumulate high pore pressure, such as the newly developed quaternary layers (Sumer, 2014). It has also been reported that residual mechanism is more important than oscillatory mechanism in the cases of large wave loading (Jeng and Seymour, 2007). Such soil will experience plastic behaviours like contraction and strain-softening under 
repeated disturbance (Seed and Rahman, 1978), and therefore is not suitable as foundations for offshore structures. However, due to its wide distribution in the coastal areas, without other better options, coastal engineers have to reveal the response mechanism of such a seabed in the marine environment and provide predictions and suggestions for the design, construction and maintenance of structures that built on this soil.

To deal with the non-linear and permanent deformation of the plastic soil behaviours, in particular to describe pore pressure accumulation and simultaneously considering dissipation, requires the implementation of an appropriate constitutive law in the numerical model and involves a more complex mathematical process. To date, a few studies for the residual seabed response around breakwaters are available in the literature. Among these, Zhao and Jeng (2015) developed an inelastic model to investigate the wave-induced accumulation of residual pore pressure within a sloping seabed in the vicinity of breakwater. They considered the seabed foundation as an inelastic medium and used the phase-resolved absolute shear stress as the source term for the build-up of pore pressures. Later, Ye et al. (2015) investigated the plastic soil behaviour around a composite breakwater and revealed the mechanism of pore water pressure accumulation within the Nevada sand seabed foundation. Considering the principal stress rotation, Zhao et al. (2017) developed another two-dimensional model to study the foundation instability for a loose sandy seabed around caisson-type breakwater under combined wave and current loading. However, these studies have been limited to two-dimensional case (Zhao and Jeng, 2015; Zhao et al., 2017; Ye et al., 2015).

In this paper, by using a 3D numerical model, we investigate the dynamic response and stability of offshore detached breakwater foundations composed of loose sand soil, whose mechanical properties are poor and vulnerable to the long-term ocean loading. The effects of a perpendicular longshore current on the flow regime and subsequent liquefaction potential of seabed foundation are considered. Finally, the susceptibility of both elastic and plastic seabed foundation to liquefaction with different wave, current, soil parameters settings is examined by a parametric study.

\section{Theoretical model}

\subsection{Problem definition}

Figure 1 shows the configuration of the computational domain for the present study. This includes: a seabed foundation is $95 \mathrm{~m} \times 80 \mathrm{~m} \times 12 \mathrm{~m}$; three breakwaters that are paralleled to the shoreline sit on the middle of the seabed foundation with width equals to $5 \mathrm{~m}$ and height equals to $5.6 \mathrm{~m}$. The length of the breakwaters on two sides is $10 \mathrm{~m}$ and $20 \mathrm{~m}$ for the middle breakwater. The gap between the breakwaters is $20 \mathrm{~m}$. The stability of the porous seabed foundation in the vicinity of the offshore detached breakwaters is examined using a 3D numerical model, which consists of two sub-models and a one-way integrated module. A description of the theoretical model will be introduced in the following sections. 


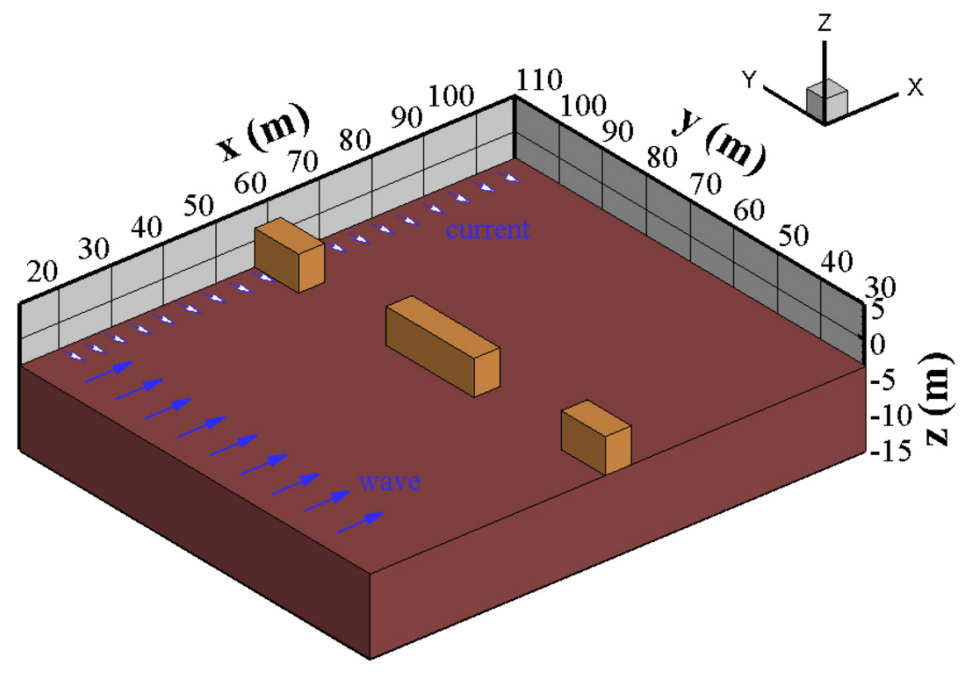

Figure 1: The computational domain of the present study.

$$
\begin{aligned}
& \frac{\partial\left\langle u_{i}\right\rangle}{\partial x_{i}}=0, \\
& \frac{\partial \rho\left\langle u_{i}\right\rangle}{\partial t}+\frac{\partial}{\partial x_{j}}\left[\frac{1}{\phi} \rho\left\langle u_{i}\right\rangle\left\langle u_{j}\right\rangle\right]=-\phi \frac{\partial\left\langle p^{*}\right\rangle^{f}}{\partial x_{i}}+\phi \rho g_{i}+\frac{\partial}{\partial x_{j}}\left[\mu_{e f f} \frac{\partial\left\langle u_{i}\right\rangle}{\partial x_{j}}\right]-[C T],
\end{aligned}
$$

89 generated by the porous material, which is calculated by, 


$$
[C T]=A\left\langle u_{i}\right\rangle+B|\langle u\rangle|\left\langle u_{i}\right\rangle+0.34 \frac{\partial\left\langle u_{i}\right\rangle}{\partial t},
$$

90

where three terms in the right-hand-side of (3) correspond to the linear, non-linear and unsteady flow within the porous media, respectively. In the present model, the Van Gent (1995)'s formulas are employed to determine coefficients $A$ and $B$,

$$
A=E_{1} \frac{(1-\phi)^{3}}{\phi^{2}} \frac{\mu}{D_{50}^{2}}, \quad \text { and } \quad B=E_{2}\left(1+\frac{7.5}{K C}\right) \frac{1-\phi}{\phi^{2}} \frac{\rho}{D_{50}}
$$

where $D_{50}$ is the medium diameter of grains; $E_{2}$ and $E_{1}$ are parameters to characterise the friction terms, which can be calibrated using physical test results based on the porous material properties and flow regime. In this study, $E_{1}=50$ and $E_{2}=1.2$ are applied, as suggested by Higuera (2015); $K C$ denotes the Keulegan-Carpenter number, as calculated as:

$$
K C=\frac{u_{m} T_{0}}{\phi D_{50}}
$$

in which $T_{0}$ is the wave period and $u_{m}$ is the maximum oscillating velocity.

The interactions between waves, currents and breakwaters generally involves in a significant amount of turbulence in the vicinity of the structures. To solve the governing equations (1)-(2), the $k-\epsilon$ turbulence model is employed,

$$
v_{t u r b}=\frac{C_{\mu} \kappa^{2}}{\epsilon}
$$

where $\kappa$ is the turbulence kinetic energy; and $\epsilon$ is the turbulence energy dissipation rate; $C_{\mu}$ is an empirical constant, usually the value of $C_{\mu}$ is 0.09 . Similar to the governing equations, the implementation of the $k-\epsilon$ model involves a volume-averaged procedure in order to account for the turbulence enhancement within the porous media (Higuera, 2015). The final expression of volume-averaged $k-\epsilon$ model for the porous flow inside porous medium is:

$$
\frac{\partial\langle k\rangle}{\partial t}+\frac{\left\langle\bar{u}_{f i}\right\rangle}{\phi} \frac{\partial\langle k\rangle}{\partial x_{j}}=\frac{\partial}{\partial x_{j}}\left[\left(\frac{\left\langle v_{t u r b}\right\rangle}{\sigma_{k}}+v\right) \frac{\partial\langle k\rangle}{\partial x_{j}}\right]-\frac{\left\langle\overline{u_{f i}^{\prime} u_{f i}^{\prime}}\right\rangle}{\phi} \frac{\partial\left\langle\bar{u}_{f i}\right\rangle}{\partial x_{j}}-\langle\epsilon\rangle+\phi \epsilon_{\infty},
$$




$$
\frac{\partial\langle\epsilon\rangle}{\partial t}+\frac{\left\langle\bar{u}_{f i}\right\rangle}{\phi} \frac{\partial \epsilon}{\partial x_{j}}=\frac{\partial}{\partial x_{j}}\left[\left(\frac{\left\langle v_{t u r b}\right\rangle}{\sigma_{\epsilon}}+v\right) \frac{\partial \epsilon}{\partial x_{j}}\right]-C_{\epsilon 1} \frac{\langle\epsilon\rangle}{\phi\langle k\rangle}\left\langle\overline{u_{f i}^{\prime} u_{f i}^{\prime}}\right\rangle \frac{\partial\left\langle\bar{u}_{f i}\right\rangle}{\partial x_{j}}-C_{\epsilon 2} \frac{\langle\epsilon\rangle^{2}}{k}+\phi C_{\epsilon 2} \frac{\epsilon_{\infty}^{2}}{k_{\infty}} .
$$

where the constants are empirically determined as: $C_{\epsilon 1}=1.44 ; C_{\epsilon 2}=1.92 ; \sigma_{k}=1.0 ; \sigma_{\epsilon}=1.3$. $\epsilon_{\infty}$ and $k_{\infty}$ can be defined as:

$$
\epsilon_{\infty}=39.0 \frac{(1-\phi)^{2.5}}{\phi}\left(\left\langle\bar{u}_{1}\right\rangle^{2}+\left\langle\bar{u}_{2}\right\rangle^{2}\right)^{1.5} \frac{1}{d_{50}}, \quad \text { and } \quad k_{\infty}=3.7 \frac{1-\phi}{\sqrt{\phi}}\left(\left\langle\bar{u}_{1}\right\rangle^{2}+\left\langle\bar{u}_{2}\right\rangle^{2}\right) .
$$

To simulate the wave-current interactions around the offshore structure, several boundary conditions are introduced. The inlet boundary condition allows for different wave generation models as well as applying the steady current flow by setting the values of velocity and Volume of Fluid function. The active wave absorption is adopted for the outlet boundary conditions to prevent the reflection of waves and currents from the boundary. As shown in Figure 1, the inlet boundary condition is set at the left and top boundary for the wave and longshore current generation respectively with the active absorption boundary condition at the corresponding opposite boundaries.

\subsection{Seabed sub-model}

The three-dimensional seabed sub-model is developed within the framework of Finite Element Method and integrated with the flow sub-model through an integration module. The soil-pore fluid interaction in a porous seabed is governed by by the $u-p$ approximations proposed by Zienkiewicz et al. (1980). The $u-p$ approximations comprise equilibrium equation and continuity equation, which can be expressed as,

$$
\begin{aligned}
& \frac{\partial \sigma_{x}^{\prime}}{\partial x}+\frac{\partial \tau_{x y}}{\partial y}+\frac{\partial \tau_{x z}}{\partial z}=-\frac{\partial p_{s}}{\partial x}+\rho^{\prime} \frac{\partial^{2} u_{s}}{\partial t^{2}}, \\
& \frac{\partial \tau_{x y}}{\partial x}+\frac{\partial \sigma_{y}^{\prime}}{\partial y}+\frac{\partial \tau_{y z}}{\partial z}=-\frac{\partial p_{s}}{\partial y}+\rho^{\prime} \frac{\partial^{2} v_{s}}{\partial t^{2}}, \\
& \frac{\partial \tau_{x z}}{\partial x}+\frac{\partial \tau_{y z}}{\partial y}+\frac{\partial \sigma_{z}^{\prime}}{\partial z}+\rho^{\prime} g=-\frac{\partial p_{s}}{\partial z}+\rho^{\prime} \frac{\partial^{2} w_{s}}{\partial t^{2}}, \\
& k_{s} \nabla^{2} p_{s}-\gamma_{w} n_{s} \beta \frac{\partial p_{s}}{\partial t}+k_{s} \rho_{f} \frac{\partial^{2} \epsilon_{s}}{\partial t^{2}}=\gamma_{w} \frac{\partial \epsilon_{s}}{\partial t},
\end{aligned}
$$




$$
\beta=\frac{1}{K_{f}}+\frac{1-S_{r}}{p_{w 0}}, \quad \text { and } \quad \epsilon_{s}=\frac{\partial u_{s}}{\partial x}+\frac{\partial v_{s}}{\partial y}+\frac{\partial w_{s}}{\partial z},
$$

where $\sigma_{i}^{\prime}$ is the effective normal stress; $\tau_{i j}$ denotes the shear stress; $p_{s}$ is pore pressure; $u_{s}, v_{s}, w_{s}$ are soil displacements; $n_{s}$ is soil porosity; $\rho^{\prime}$ denotes average density of soil $\left(\rho^{\prime}=\rho_{f} n_{s}+\rho_{s}\left(1-n_{s}\right)\right.$, in which $\rho_{f}$ is fluid density and $\rho_{s}=$ soil density); $\gamma_{w}$ is the unit weight of water; $k_{s}$ is soil permeability; $\beta$ and $\epsilon_{s}$ are the pore water compressibility and volume strain respectively (calculated as (14), in which where $S_{r}$ represents the degree of saturation; $p_{w 0}$ is the absolute static pressure; $K_{f}$ denotes bulk modulus of pore fluid, whose value is suggested by Yamamoto et al. (1978) as $1.95 \times 10^{9} \mathrm{~N} / \mathrm{m}^{2}$ for pore water).

The stress-strain relationship for the seabed is determined by the soil constitutive models incorporated within the seabed model. For the elastic seabed with reversible strain, the relationship is expressed as:

$$
d \sigma_{i j}^{\prime}=D_{i j k l}^{e} d \epsilon_{k l}^{e}, \quad \text { and } \quad D_{i j k l}^{e}=\lambda^{\prime} \delta_{i j} \delta_{k l}+2 G \delta_{i k} \delta_{j l} \text {, }
$$

in which $d \sigma_{i j}^{\prime}, d \epsilon_{k l}^{e}$ are the effective stresses increment and strain increment; $D_{i j k l}^{e}$ is the elastic constitutive matrix; and $\lambda^{\prime}$ denotes the Lame's constant; $G$ represents the shear modulus.

For an elasto-plastic seabed with irreversible soil contraction, the Pastor-Zienkiewicz-Mark-III (PZIII) model (Pastor et al., 1990) is adopted, whose strain increment is decomposed into two components:

$$
d \epsilon_{i j}=d \epsilon_{i j}^{p}+d \epsilon_{i j}^{e},
$$

where $d \epsilon_{i j}^{p}$ is the plastic component accounting for the irreversible strain; and $d \epsilon_{i j}^{e}$ is the elastic component accounting for the reversible strain. The elastic strain is still governed by the Hooke's law as Equation (15). The elastic volumetric strain-rate and deviatoric strain-rate are considered separately as:

$$
\dot{\epsilon}_{v}^{e}=\frac{\dot{p}^{\prime}}{K_{e v}} \quad \text { and } \quad \dot{\epsilon}_{s}^{e}=\frac{\dot{q}}{G_{e s}}
$$

where $\dot{\epsilon}_{v}^{e}$ and $\dot{\epsilon}_{s}^{e}$ are the elastic volumetric strain and deviatoric strain respectively; $K_{e v}$ is the soil bulk modulus; $G_{e s}$ denotes shear modulus; $p^{\prime}$ represents the mean effective stress and $q$ is deviatoric stress,

$$
p^{\prime}=\frac{1}{3}\left(\sigma_{x}^{\prime}+\sigma_{y}^{\prime}+\sigma_{z}^{\prime}\right), \quad \text { and } \quad q=\sqrt{\frac{\left(\sigma_{x}^{\prime}-\sigma_{y}^{\prime}\right)^{2}+\left(\sigma_{x}^{\prime}-\sigma_{z}^{\prime}\right)^{2}+\left(\sigma_{y}^{\prime}-\sigma_{z}^{\prime}\right)^{2}+6\left(\tau_{x y}^{2}+\tau_{y z}^{2}+\tau_{x z}^{2}\right)}{3}} \text {. }
$$




$$
m_{i j}=\frac{\left(\frac{\partial g}{\partial \sigma_{i j}^{\prime}}\right)}{\left\|\frac{\partial g}{\partial \sigma_{i j}^{\prime}}\right\|}, \quad \text { and } \quad n_{i j}=\frac{\left(\frac{\partial f}{\partial \sigma_{i j}^{\prime}}\right)}{\left\|\frac{\partial f}{\partial \sigma_{i j}^{\prime}}\right\|}
$$

145 where $\left\|\frac{\partial g}{\partial \sigma_{i j}^{\prime}}\right\|$ and $\left\|\frac{\partial f}{\partial \sigma_{i j}^{\prime}}\right\|$ are the norm of the tensor $\frac{\partial g}{\partial \sigma_{i j}^{\prime}}$ and $\frac{\partial f}{\partial \sigma_{i j}^{\prime}}$, respectively.

${ }_{146}$ The loading and unloading status are defined by following expressions,

$$
\left\{\begin{array}{lll}
\text { if } & n_{i j} d \sigma_{i j}^{\prime}>0, & \text { Loading } \\
\text { if } & n_{i j} d \sigma_{i j}^{\prime}=0, & \text { Neutral-loading } \\
\text { if } & n_{i j} d \sigma_{i j}^{\prime}<0, & \text { Unloading }
\end{array}\right.
$$

147 A smoothed Mohr-Coulomb criterion is employed in the PZIII model to generalise the critical state line to three-

$$
K_{e v}=K_{e v 0} \frac{p^{\prime}}{p_{0}^{\prime}} \quad \text { and } \quad G_{e s}=G_{e s 0} \frac{p^{\prime}}{p_{0}^{\prime}}
$$

where $p_{0}^{\prime}$ is the mean effective stress used to measure the elastic parameter of soil; $K_{e v 0}$ and $G_{e s 0}$ are the elastic bulk modulus and shear modulus of soil under $p_{0}^{\prime}$.

The the plastic potential function $g$ and loading functions $f$ are defined as:

$$
g=q-M_{g} p^{\prime}\left(1+\frac{1}{\alpha_{g}}\right)\left[1-\left(\frac{p^{\prime}}{p_{g}^{\prime}}\right)^{\alpha_{g}}\right]=0, \quad \text { and } \quad f=q-M_{f} p^{\prime}\left(1+\frac{1}{\alpha_{f}}\right)\left[1-\left(\frac{p^{\prime}}{p_{f}^{\prime}}\right)^{\alpha_{f}}\right]=0
$$

$$
\frac{M_{f}}{M_{g}}=D_{r}
$$

148 dimensional stress state, 


$$
M_{g}=\frac{6 \sin \phi^{\prime}}{3-\sin \phi^{\prime} \sin 3 \theta},
$$

where $\theta$ is the Lode's angle and $\phi^{\prime}$ is the residual friction angle of sand measured when $\theta=30^{\circ}$.

In summary, the plastic strain increment can be defined as:

$$
d \epsilon_{i j}^{p}=\frac{1}{H_{L / U}} m_{i j} n_{k l} d \sigma_{k l}^{\prime},
$$

in which $H_{L / U}$ stands for the plastic modulus for the loading or unloading stage:

$$
\begin{aligned}
& H_{L}=H_{0} p^{\prime}\left(1-\frac{q / p^{\prime}}{\eta_{f}}\right)^{4}\left[1-\frac{q / p^{\prime}}{M_{g}}+\beta_{0} \beta_{1} \exp \left(-\beta_{0} \xi\right)\right]\left(\frac{q / p^{\prime}}{\eta_{\max }}\right)^{-\gamma_{D M}}, \\
& H_{U}= \begin{cases}H_{U 0}\left(\frac{M_{g}}{\eta_{u}}\right)^{\gamma_{U}} & \text { for } \quad\left|\frac{M_{g}}{\eta_{u}}\right|>1 \\
H_{U 0} & \text { for } \quad\left|\frac{M_{g}}{\eta_{u}}\right| \leq 1\end{cases}
\end{aligned}
$$

where $H_{0}$ is a parameter that scales the plastic modulus; $H_{U 0}$ is the parameter which scale the plastic modulus; $\eta_{u}$ is the stress ratio from which the unloading takes place and $\gamma_{U}$ is the material constant controlling the influence of it.

In summary, the constitutive relationship for the elastoplastic seabed foundation can be expressed as:

$$
d \epsilon_{i j}=C_{i j k l}^{e} d \sigma_{k l}^{\prime e}+\frac{1}{H_{L / U}} m_{i j} n_{k l} d \sigma_{k l}^{\prime},
$$

where $C_{i j k l}^{e}$ is the elastic compliance tensor.

\subsection{Integration of flow and seabed sub-models}

Figure 2 illustrates the computational procedure of the present study, including three parts: pre-processing, numerical simulation and post-processing. The integrated process between the flow and seabed sub-models is reflected in the content framed by the dotted box, in which a one-way coupling algorithm is adopted through the pressure continuity on the common waves-seabed interfaces. As shown in Figure 2, the work flow of this study is: in the pre-processing stage, the integral 3D numerical model is established by configuring the computational domain, dividing the mesh grids, setting the time interval scheme, determining the initial fields and inputting 
various model parameters. After accomplishing the process of pre-processing, the fluid motion that interacted with the offshore detached breakwaters are solved by the VARANS equations along with the $k-\epsilon$ turbulence model; at each time step, the hydrodynamic pressure at the waves-seabed interface is extracted from the flow submodel and applied to the soil model; then, using the hydrodynamic pressure at the seabed surface as the boundary condition, the soil model solves the partially dynamic Biot's ( $u-p$ approximations) equations. Depending on the different constitutive models, the elastic reversible soil behaviours and elastoplastic irreversible soil responses can be obtained. The simulation finishes when the time step reaches the prescribed total simulation time and we can obtain the main outputs include: the hydrodynamic pressure field, velocity field and volume fraction function field in the fluid domain and pore pressures, effective stresses, volumetric strain and soil displacements etc. in the solid domain. By post-processing these results, we can try to understand the mechanism of wave-current-seabedbreakwaters interactions and evaluate the liquefaction potential in the vicinity of the breakwaters.

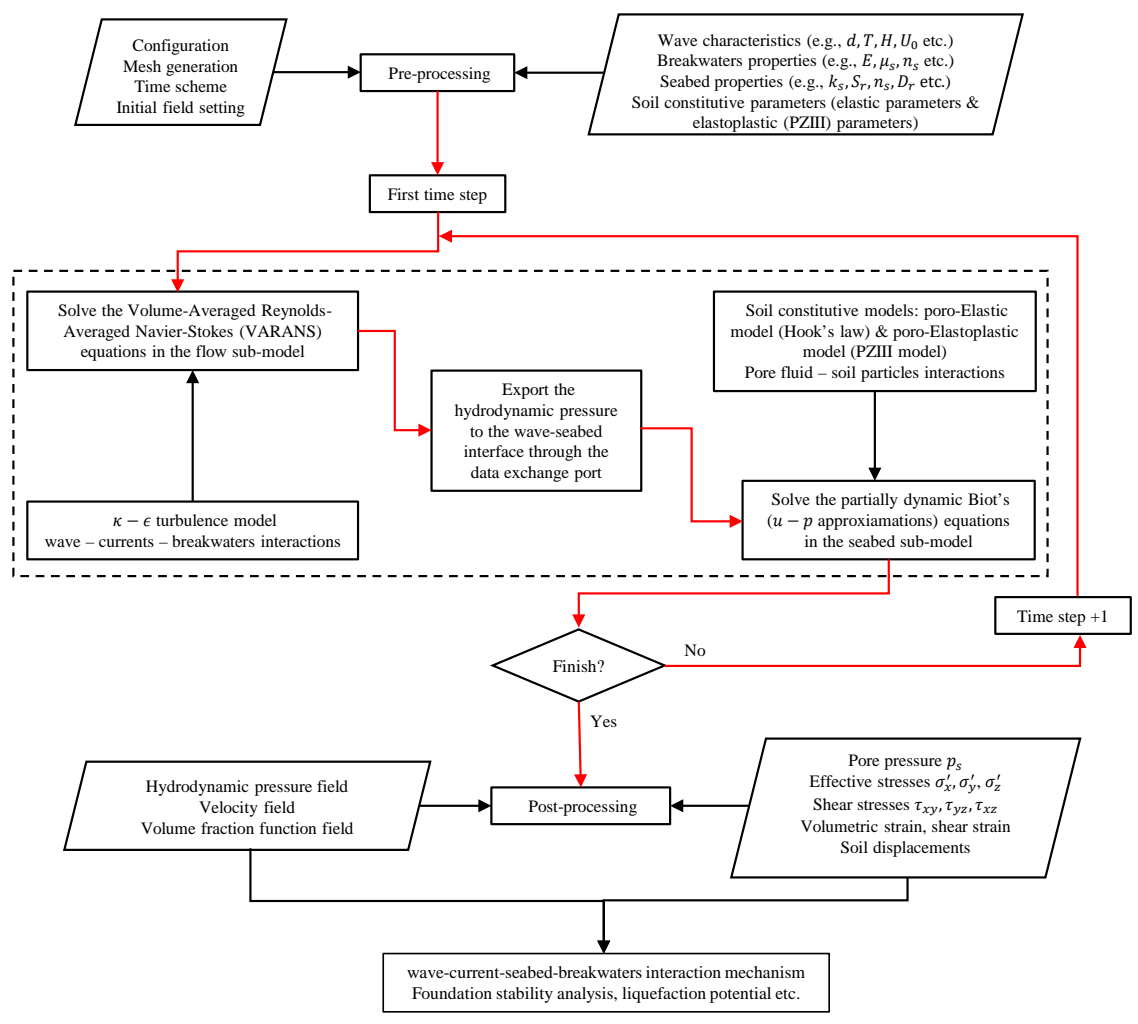

Figure 2: The coupling process of the numerical model.

\section{Model validations}

In this section, three validations of the present model are presented, including 
- Validation 1: This validation is to compare the present model with the wave flume tests (Qi et al., 2019) for the excess pore pressure in a seabed without a structure under combined wave and current loading. Note that the surface elevation obtained from the present fluid sub-model are also compared with the experimental data.

- Validation 2: The wave flume experiments (Mizutani et al., 1998) will be compared with the present model for the case with a submerged rouble mould breakwaters under wave loading.

- Validation 3: The present model is compared with the centrifugal tests for the wave-induced residual pore pressures Sassa and Sekiguchi (1999).

Note that all the above comparisons are 2D cases, because no 3D experimental data is available.

\subsection{Validation 1}

The present model was first verified by reproducing the results from Qi et al. (2019)'s wave flume tests, in which they conducted a series of flume tests to obtained the excess pore pressure in a sandy seabed under combined wave and current loading. Figure 3 is the sketch of the specially-designed flume used in Qi et al. (2019)'s tests. The flume is able to generate periodic wave and bidirectional current simultaneously. Four wave height gauges (WHGs) were utilised to measure the wave height and calculate the wave length in real time and four miniature pore pressure transducers (PPTs) were installed right below the No. III and IV WHGs to measure the wave/current induced pore pressure within the sandy seabed.

In Qi et al. (2019)'s experiments, four different current conditions together with waves were considered. Among these, the observations from run No. 1 (wave loading only) and run No. 9 (waves with a $0.25 \mathrm{~m} / \mathrm{s}$ following current) are chosen to validate the present model. All other parameters are identical in both tests: the mean water depth $(d)$ is $0.5 \mathrm{~m}$, the wave period $(T)$ is $1.2 \mathrm{~s}$, the wave height $(H)$ is $9.5 \mathrm{~cm}$. The sand used in the experiments has following properties: the mean size of grains $\left(d_{50}\right)$ is $0.38 \mathrm{~mm}$, the void ratio $(e)$ is 0.771 , the relative density $\left(D_{r}\right)$ is 0.352 and the buoyant unit weight $\left(\gamma^{\prime}\right)$ is $9.32 \mathrm{kN} / \mathrm{m}^{3}$.

Figure 4 shows the time series of surface elevation from flume observations and numerical predictions for run No. 1 (wave only) and run No. 9 (wave with a following current) at WHG III. It is found from the figure, the wave steepness (i.e., wave height/wave length) is slightly decreased due to the presence of the current. The numerical model can predict the change of surface elevation fairly well. This provides the validation of the flow model. The comparison of excess pore pressures at PPT1, PPT2 and PPT3 for run No. 1 and run No. 9 is shown in Figure 5. The numerical results are consistent with the experimental results, which indicates that the present model has the ability to accurately predict the wave-current induced seabed response. 


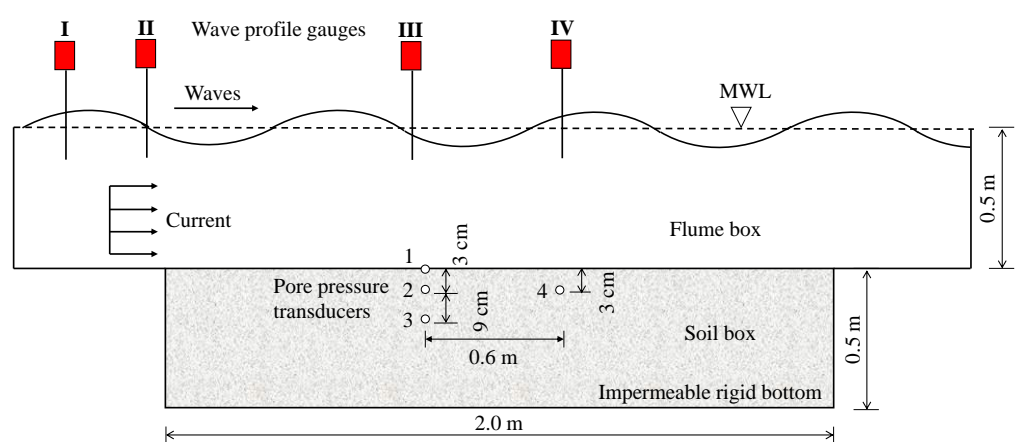

Figure 3: The sketch of Qi et al. (2019)'s flume tests.
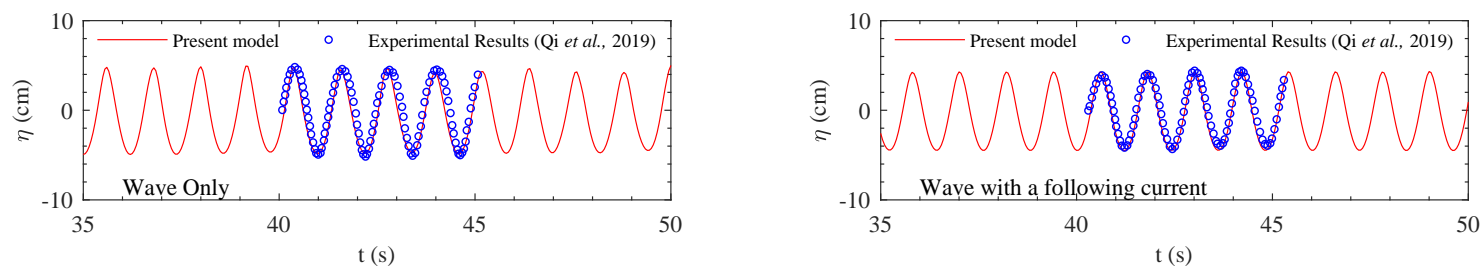

Figure 4: Comparison between the simulation results and Qi et al. (2019)'s experimental results for the surface elevation.
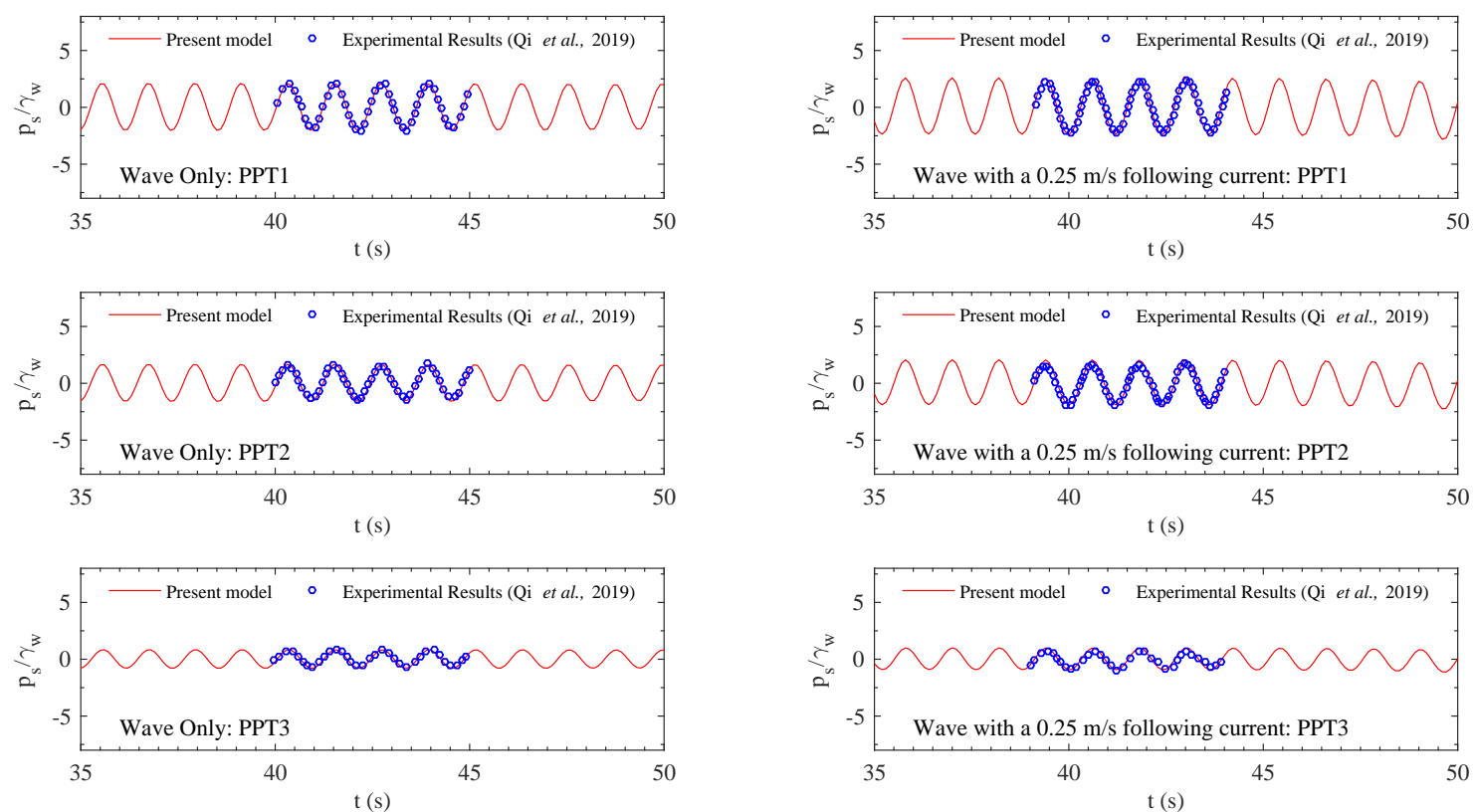

Figure 5: Comparison between the simulation results and Qi et al. (2019)'s experimental results for the excess pore pressure.

\subsection{Validation 2}

In order to investigate the dynamic interactions between nonlinear regular wave, submerged breakwater and seabed foundation, Mizutani et al. (1998) carried out a series of experiments at Nagoya University. The experimental set-up is shown in Figure 6. Results from their experiments, including the surface elevation around breakwater and pore pressure within the solid domain, are adopted to validate the present model. 


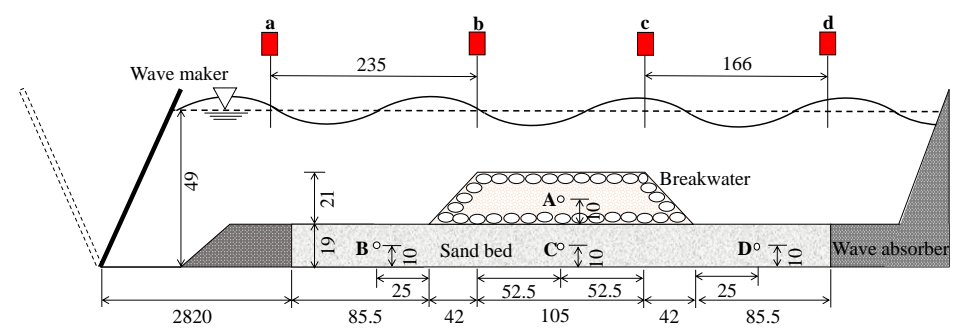

Figure 6: The set-up of Mizutani et al. (1998)'s experiments (units: cm).

Figure 7 illustrates the results of surface elevation that were measured at wave meters a, b, c and d. The predicted results agree quite well with the experimental data both for the location in front of the breakwater and behind the breakwater. As can be seen at locations behind the breakwater (location c and d), some non-linearity and wave damping occurred after interacting with the breakwater, the numerical model is able to capture these features.
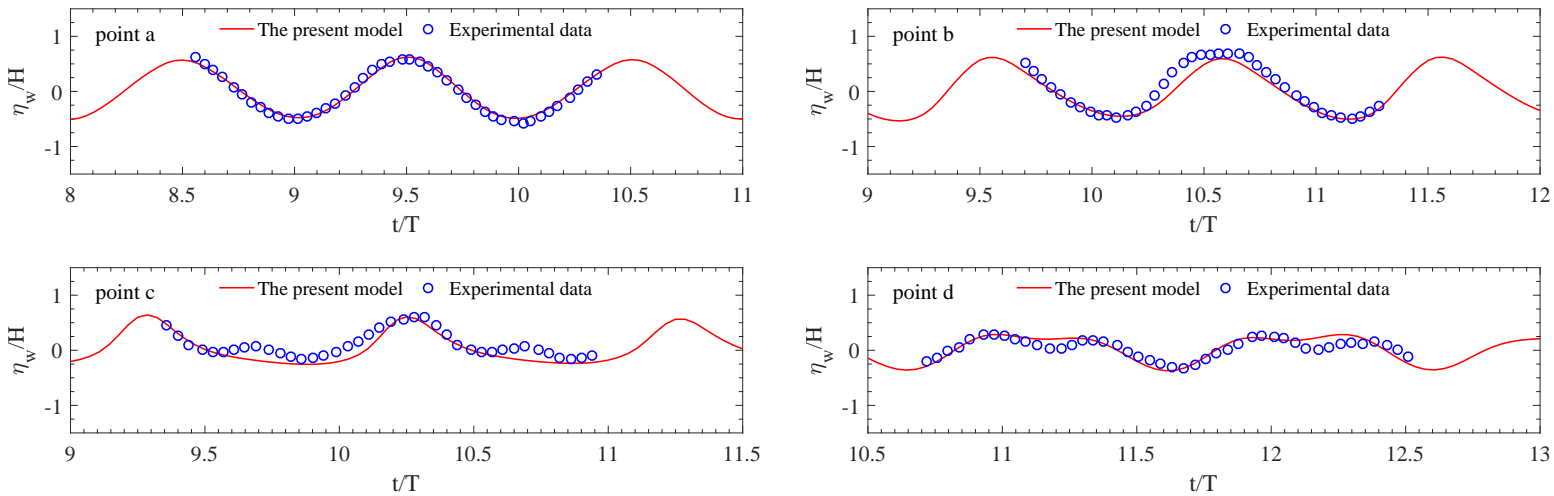

Figure 7: Comparison between the simulation results and Mizutani et al. (1998)'s experimental results for the surface elevation.

Figure 8 shows the pore pressures in the breakwater (point A) and seabed (points B, C and D). It is clear that the comparison is quite consistent which indicates that the integrated model is capable of simulating problems of waveseabed-porous breakwater interaction. It is worth noting that in order to reproduce the experimental procedure that the sand was consolidated for several days, a consolidation process was also conducted in the numerical simulation under the self-weight of breakwater and hydro-static pressure.

\subsection{Validation 3}

The third validation is to compare the residual soil response in a seabed foundation under periodic waves with the geo-centrifugal tests (Sassa and Sekiguchi, 1999). Wave test P5-1 from Sassa and Sekiguchi (1999)'s centrifuge tests is adopted to verify the present model. From the set-up of Sassa and Sekiguchi (1999)'s test (see Figure 9), a $200 \mathrm{~mm}$ wide and $100 \mathrm{~mm}$ deep seabed foundation is composed of the saturated sand. Four pore pressure sensors 

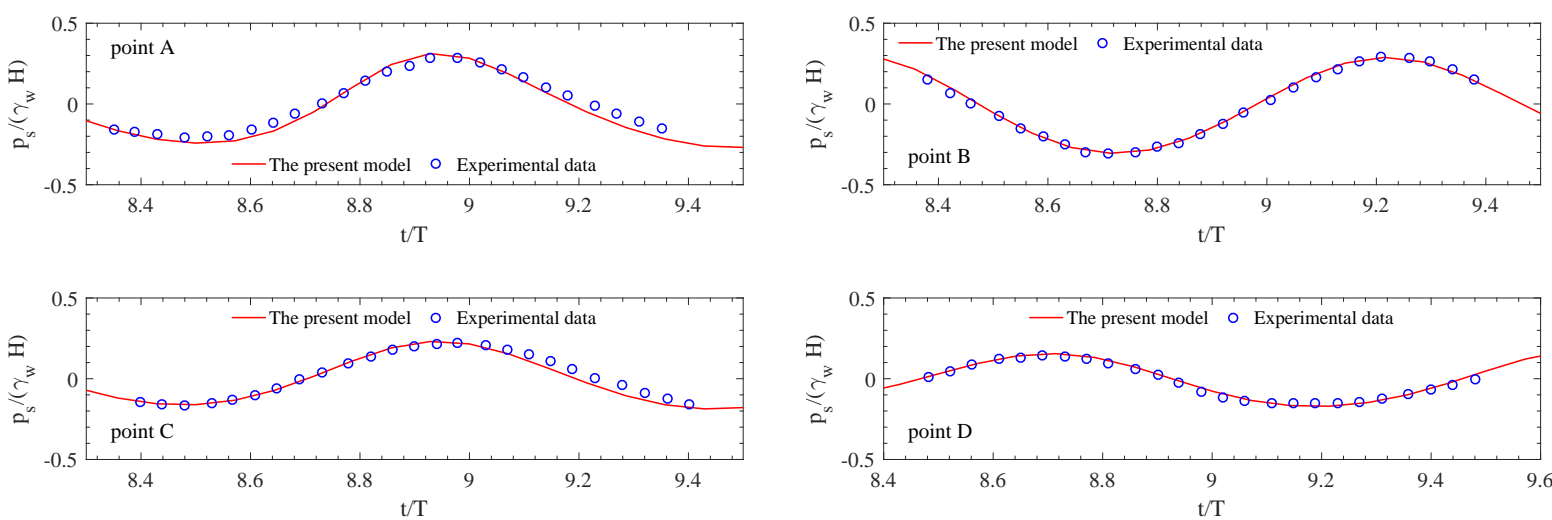

Figure 8: Comparison between the simulation results and Mizutani et al. (1998)'s experimental results for the wave induced dynamic pore pressure.

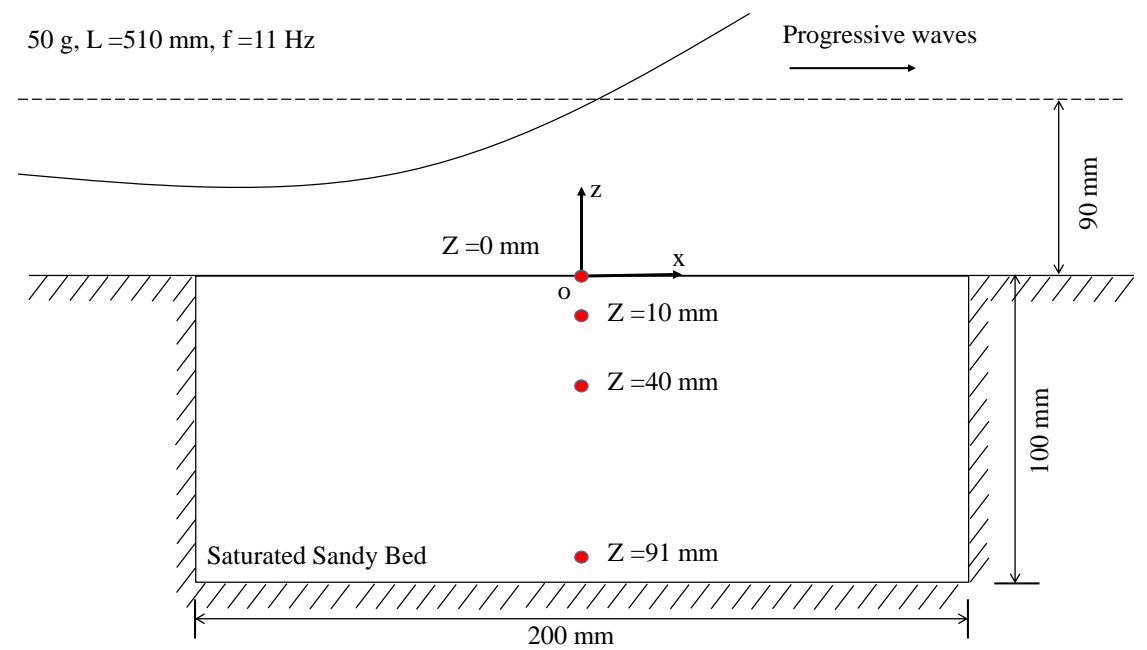

Figure 9: The sketch of the centrifuge wave test.

are installed in a vertical array in the centre line of the soil bed whose distance to the seabed surface is $0,10,40$ and $91 \mathrm{~mm}$, respectively. In order to perform a 1/50 scaled centrifugal model, a $50 \mathrm{~g}$ steady-state acceleration is applied and the fluid is used by the silicone oil with a viscosity of $50 \mathrm{cSt}$. The equivalent wave and soil parameters for $1 \mathrm{~g}$ acceleration calculated based on the scaling principal are listed in Table 1.

Figure 10 shows the comparison of the wave induced pore pressure changes in the seabed measured by four pore pressure sensors between the numerical and centrifuge test results. Note that only the residual part of centrifuge test results is presented in the figure as indicated as the blue dashed line. An overall good agreement is observed between the presented model and the centrifuge tests (Sassa and Sekiguchi, 1999). This indicates that the present model is able to predict the accumulation process of the excess pore pressure over time at different depths. 
Table 1: Parameters for validation 3.

\begin{tabular}{lc}
\hline \multicolumn{2}{c}{ Wave characteristics } \\
\hline Wave height $(H)$ & $1.7 \mathrm{~m}$ \\
Wave period $(T)$ & $4.55 \mathrm{~s}$ \\
Water depth $(d)$ & $4.5 \mathrm{~m}$ \\
\hline \multicolumn{2}{c}{ Soil characteristics } \\
\hline Poisson's ratio $\left(\mu_{s}\right)$ & 0.3 \\
Permeability $\left(k_{s}\right)$ & $1.5 \times 10^{-4} \mathrm{~m} / \mathrm{s}$ \\
Porosity $\left(n_{s}\right)$ & 0.445 \\
Degree of saturation $\left(S_{r}\right)$ & $100 \%$ \\
Relative density $\left(D_{r}\right)$ & $42 \%$ \\
\hline \multicolumn{2}{c}{ Parameters for PZIII model } \\
\hline$M_{g}$ & 1.2124 \\
$M_{f}$ & 0.75 \\
$\alpha_{f}$ & 0.1 \\
$\alpha_{g}$ & 0.1 \\
$K_{\text {evo }}$ & $660.8 \mathrm{KPa}$ \\
$G_{\text {eso }}$ & $770.0 \mathrm{kPa}$ \\
$\beta_{0}$ & 0.2 \\
$\beta_{1}$ & 2.5 \\
$p_{0}^{\prime}$ & $4.0 \mathrm{kPa}$ \\
$H_{0}$ & 700.0 \\
$H_{U 0}$ & $1000.0 \mathrm{kPa}$ \\
$\gamma_{u}$ & 6.0 \\
$\gamma_{D M}$ & 4.0 \\
\hline
\end{tabular}


Figure 10: The excess pore pressure in the sandy seabed from the simulation results and Sassa and Sekiguchi (1999)'s centrifuge test

The above validations have demonstrated the capacity of the present model in the prediction of the wave 237 (current)-induced pore pressures in a porous seabed. 


\section{Results and discussions}

\subsection{Configuration of the breakwaters and input parameters}

Figure 11 illustrates the computational domain. The waves are generated at the left boundary and propagate rightward to the breakwaters. Currents that travel at right angle to the incident waves (in negative $y$-axis direction) are to simulate the longshore currents, which commonly exist in the near shore zones. Three main zones can be identified in this study, which contain different wave/current components. Zone I is in front of the breakwaters including incident waves, reflected waves and longshore currents, zone II is between the breakwaters including only the incident and diffracted waves, while zone III is behind the breakwaters including part of the incident waves, diffracted waves and currents. Due to the different wave/current impacts, the soil behaviours should be different from zone to zone. Hence, four reference points (A, B, C and D) are selected in corresponding zones to monitor the hydrodynamic process caused by wave/current-structure interactions and soil behaviour within the seabed foundation.

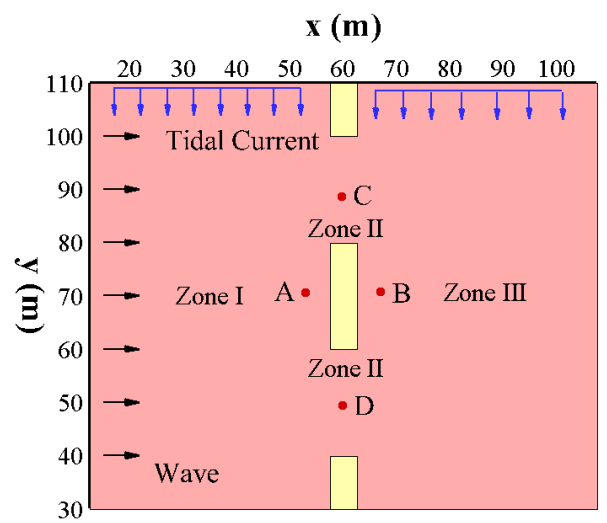

(a) Top view

$\mathbf{x}(\mathbf{m})$

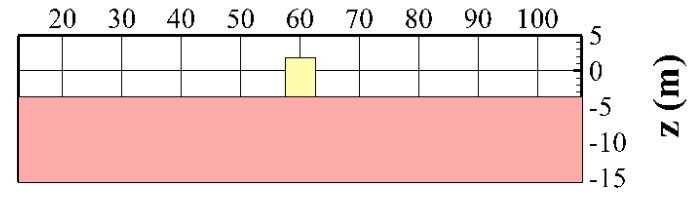

(b) Front view y (m)



(c) Side view

Figure 11: The computational domain: (a) top view, (b) front view, and (c) side view.

The seabed foundation is treated as both poro-elastic material and poro-elastoplastic material, which is simulated by the elastic and plastic constitutive models, respectively. The input parameters of the wave characteristics, properties of the breakwaters and porous seabed foundation are listed in Table 2. 
Table 2: The input parameters.




Table 3: Mesh size for the convergence tests

\begin{tabular}{l|ccc|c}
\hline Mesh scheme & $\Delta x$ & $\Delta y$ & $\Delta z$ & Cell numbers \\
\hline 1 & 0.625 & 0.625 & 0.13 & 1190991 \\
2 & 0.78 & 0.78 & 0.13 & 764679 \\
$\mathbf{3}$ & $\mathbf{1 . 0}$ & $\mathbf{1 . 0}$ & $\mathbf{0 . 1 3}$ & $\mathbf{4 6 5 2 3 1}$ \\
4 & 2.0 & 2.0 & 0.13 & 116308 \\
\hline 5 & 1.0 & 1.0 & 0.1 & 604800 \\
6 & 1.0 & 1.0 & 0.2 & 302400 \\
7 & 1.0 & 1.0 & 0.3 & 201600 \\
\hline
\end{tabular}

\subsection{Mesh convergence}

In this section, the grid resolution sensitivity is tested through a set of mesh schemes (see Table 3 ) by comparing the free surface elevation and hydrodynamic pressure at a point near the inlet of the computational domain. The mesh scheme No. 1-4 are designed to assess the impact of mesh size in the $x$ - and $y$-directions ( $\Delta x$ and $\Delta y$ ), which are set as identical in this study; while the mesh scheme No. 3, 5-7 are to evaluate sensitivity of $\Delta z$. In general, a denser mesh resolution can greatly increases the computational cost, which requires longer simulation time and larger computing memory. Therefore, it is important to find a suitable mesh size, which will not waste too much computing resource, but also provide a fairly accurate prediction.

Figure 12 shows the changes of free surface elevation and hydrodynamic pressure over time for different mesh schemes. The grid size increases gradually as the legends from left to right in both $x-(y-)$ and $z$ - directions (e.g., $\mathrm{m} 1<\mathrm{m} 2<\mathrm{m} 3<\mathrm{m} 4 ; \mathrm{m} 5<\mathrm{m} 3<\mathrm{m} 6<\mathrm{m} 7$ ). Taking into account the economy of the computing resources and accuracy of the simulation results, mesh scheme No.3 is the best for the current condition, whose $\Delta x=\Delta y=1.0$ $\mathrm{m}, \Delta z=0.13 \mathrm{~m}$ and total cell number is 465231 . According to the shape of surface elevation and hydrodynamic pressure curve, $\mathrm{m} 4$ is clearly not convergent. Although there are still slight differences between $\mathrm{m} 3$ and $\mathrm{m} 1 \& 2$ in the $x-(y-)$ direction, $\mathrm{m} 3$ is still the best choice, considering that the exponentially increasing amount of computation makes only minimal difference.

\subsection{Hydrodynamics around offshore detached breakwaters}

Figure 13 illustrates the impact of longshore currents to flow field around breakwaters. The velocity vectors around the offshore detached breakwater at water depth of $-2 \mathrm{~m}$ are presented. The longshore currents commonly exist in the nearshore zone that move parallel to the shoreline. It is clear that the presence of currents has a significant impact on the velocity field. At the beginning of the simulation when the first wave has not reached the breakwater (e.g., $t=4.5 \mathrm{~s}$ ), a steady lateral velocity field has formed within the whole region under the condition where currents are existing (Figures $13 \mathrm{a} \& \mathrm{c}$ ). The averaged velocity for two cases are $0.164 \mathrm{~m} / \mathrm{s}$ and $0.632 \mathrm{~m} / \mathrm{s}$, respectively. The presence of the longshore currents significantly increase the velocity field in the vicinity of 

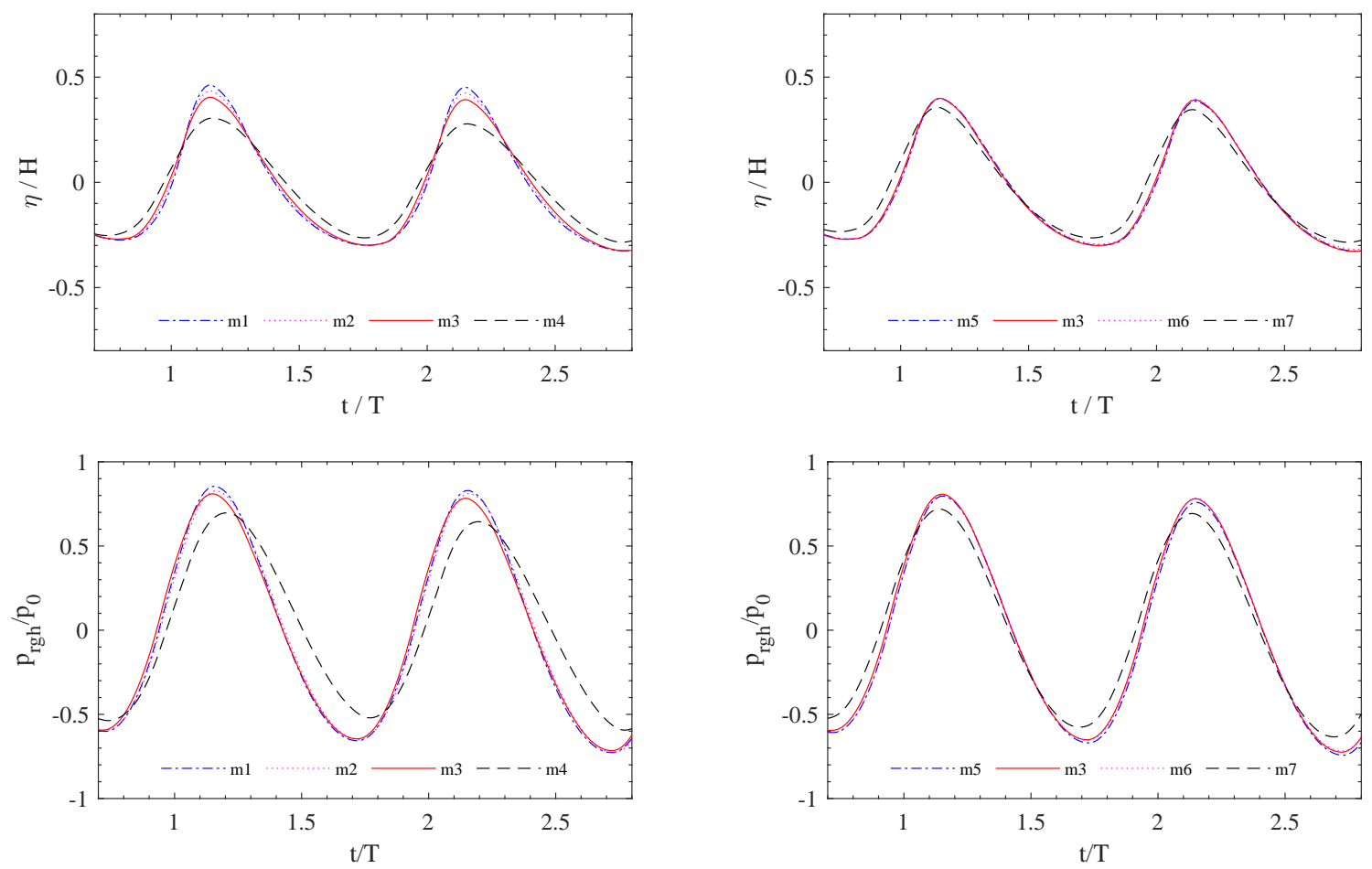

Figure 12: Mesh convergence analysis with wave condition as: $H=2.4 \mathrm{~m}, T=4.5 \mathrm{~s}, d=3.6 \mathrm{~m}$.

breakwaters. When time reaches $100 \mathrm{~s}$, the impact is even more obvious, as shown in Figures 13 b\&d. For the condition with wave loading only (Figures $13 \mathrm{a} \& \mathrm{~b}$ ), the pattern of flow field is close to symmetry due to symmetry of computational domain. The waves in front of the three breakwaters have a higher velocity because of the superimposition of the reflected waves. Non-linear interactions between the fluid and the structures can be clearly observed, some symmetrical vortexes form behind the breakwater gaps. While for the condition with perpendicular longshore currents, the turbulent wave motion is more obvious. The velocity of the waves tend to be accelerated in the whole domain and the direction of the waves tend to be push to the downward by the lateral currents.

Figure 14 illustrates the time series of water surface elevation at locations A, B, C and D (refer to Figure 11) in different zones for case with longshore currents and case with waves only. Figures show that, for the wave height, location A in zone I is the largest, followed by location C \& D in Zone II and location B in zone III. It is because of the blockage effect of the breakwaters, the incident and reflected waves were superposed in front of the breakwaters, while there are only diffracted waves behind the breakwaters. The surface elevations at location C \& D are completely coincident in the case with only waves due to the symmetry. However, the presence of the currents can change this situation, it makes the wave crest at location D more sharp and a deviation of free water surface elevation between location $\mathrm{C}$ and $\mathrm{D}$ can be observed. The same effects can be observed at location $\mathrm{A} \&$ 


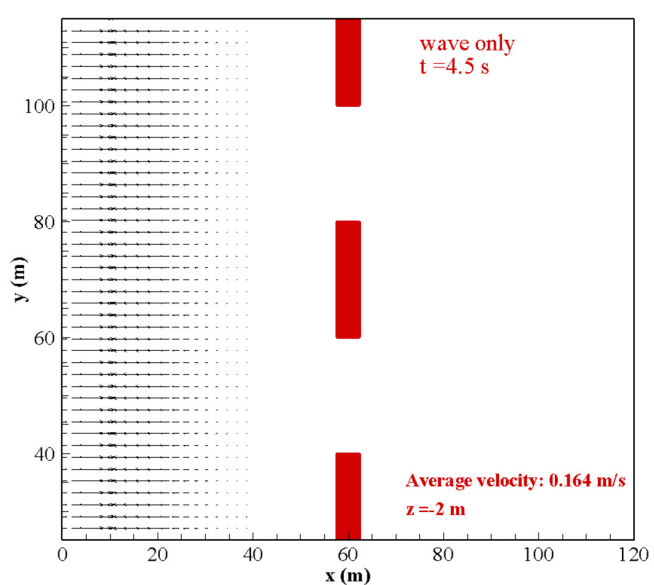

(a) $t=4.5 \mathrm{~s}$ (wave only)

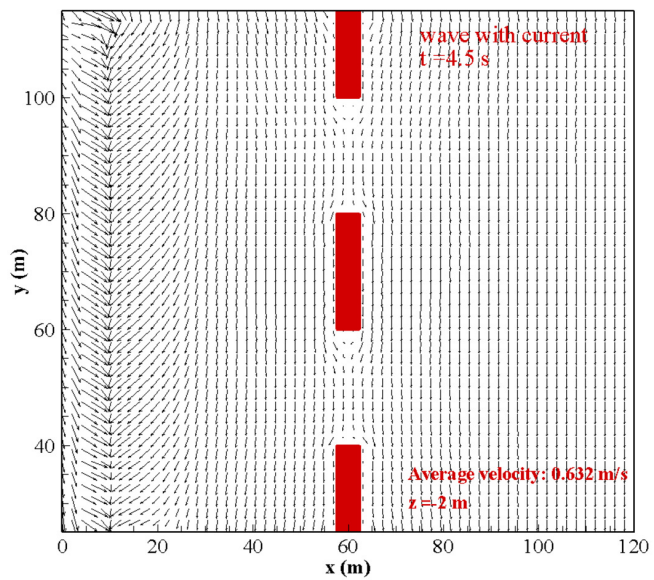

(c) $t=4.5 \mathrm{~s}$ (wave with current)

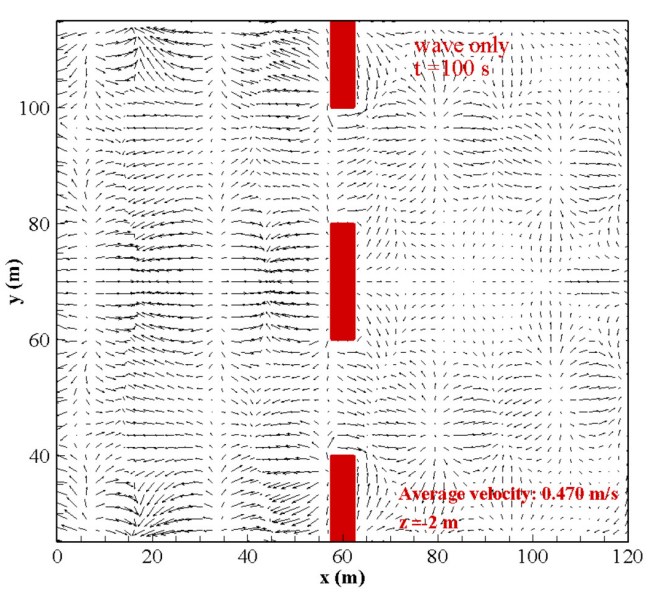

(b) $t=100 \mathrm{~s}$ (wave only)

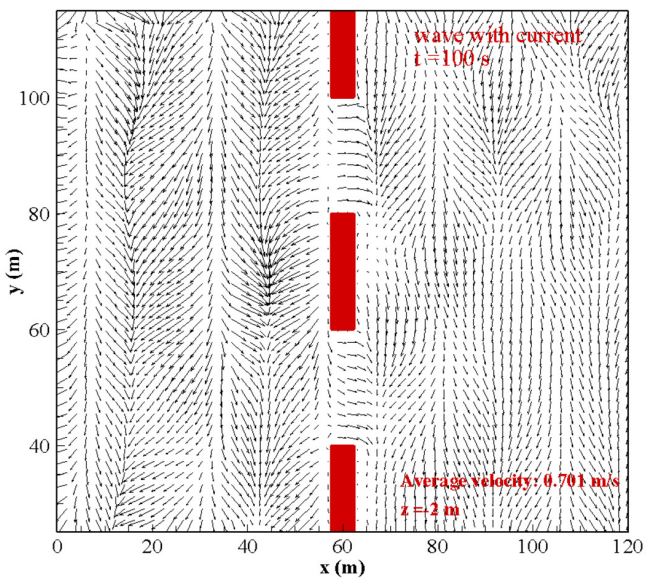

(d) $t=100 \mathrm{~s}$ (wave with current)

Figure 13: The distribution of the velocity vector of flow field around breakwaters under conditions without currents and with currents at (a) \& (b) $\mathrm{t}=4.5 \mathrm{~s}$ and (c) \& (d) $100 \mathrm{~s}$.

$\mathrm{B}$, the shape of the waves is altered by longshore current. This may further has impacts on the soil response in the seabed foundation and structure stability.
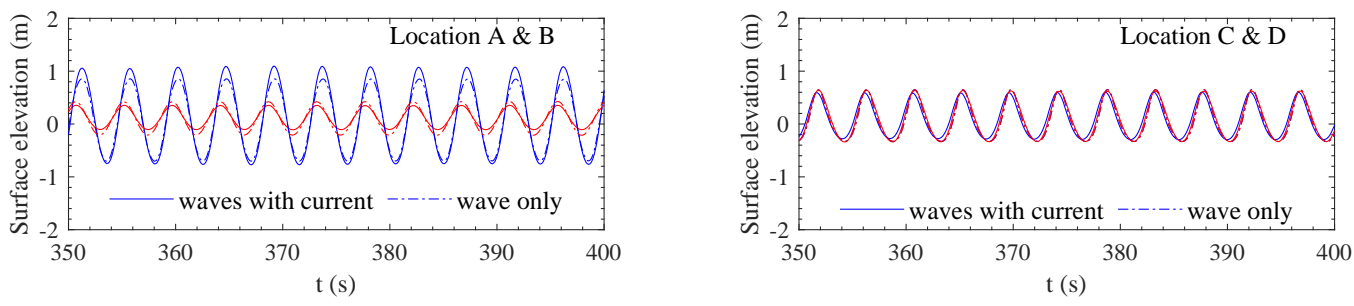

Figure 14: The surface elevation along time at location A, B, C \& D in the cases with currents and without currents. 


\subsection{Consolidation process of the seabed foundation}

The construction of marine gravity structures like breakwaters will consolidate the seabed foundation, particularly for the loose soil that can be affected significantly. When the breakwater is built on the seabed, as the excess pore pressure dissipates, the self-weight of the structure gradually transfers from pore water to the soil particle, the seabed eventually reaches a new stress state, which is named as the initial condition for the following dynamic analysis of wave/current-seabed-breakwater interactions. During this process, only the self-weight of breakwaters and hydro-static pressure from depth of water are considered without including the dynamic loading from wave and current. The seabed will be liquefied when the averaged dynamic effective stresses is greater than the averaged initial effective stresses (Tsai, 1995). Since the consolidation caused by breakwaters can increase the initial effective stresses, an overestimation of liquefaction can be assessed in the region below the breakwaters when the consolidation has not been taken into consideration.

Figure 15 displays the distributions of the pore pressure $\left(p_{s}\right)$, effective stresses $\left(\sigma_{x}^{\prime}, \sigma_{y}^{\prime}\right.$ and $\left.\sigma_{z}^{\prime}\right)$ and shear stresses $\left(\tau_{x y}, \tau_{y z}\right.$ and $\left.\tau_{x z}\right)$ within the seabed foundation after consolidation process. The pore pressure is uniformly distributed and appears as layered, indicating that the excess pore pressure disappeared and the consolidation process has completed. The figure demonstrates that the breakwaters significantly affect the stress field of the surrounding soil. $\sigma_{x}^{\prime}, \sigma_{y}^{\prime}$ and $\sigma_{z}^{\prime}$ all have a significant increase in the region under or near three breakwaters due to the compression effect, especially $\sigma_{z}^{\prime}$ whose maximum value reached $120 \mathrm{kPa}$. The increase of initial effective stresses will cause the threshold of liquefaction to increase, in other words, ignoring the consolidation process would overestimate the liquefaction potential around breakwaters. The concentration zones of $\tau_{x y}$ and $\tau_{y z}$ appear at the bottom corner of three breakwaters. Whereas, $\tau_{x z}$ distributes in the whole zone under the breakwaters and its magnitude is the largest which reaches up to $35 \mathrm{kPa}$. It is also found that the distribution of the effective stresses has not been affected in the region far away from the breakwaters, still showing uniformly layered pattern as the condition when there are no structures.

\section{5. dynamic soil responses around the offshore detached breakwaters}

Different from the elastic soil, the behaviour of elastoplastic soil depends not only on the initial and final stress state, but also on the path it has gone through to reach the final state. The stress path can effectively describe the mechanical properties of the elastoplastic seabed under the long-term cyclic waves, thus reflecting the strength variation and the possibility of liquefaction of the seabed. Figure 16 shows the effective stress paths at four reference points (location A, B, C \& D in Figure 11) at depth of $0.2 h$ in the seabed foundation under cyclic waves. It is found that the mean effective stress $p^{\prime}$ and the deviatoric stress $q$ both drop quickly at all locations 


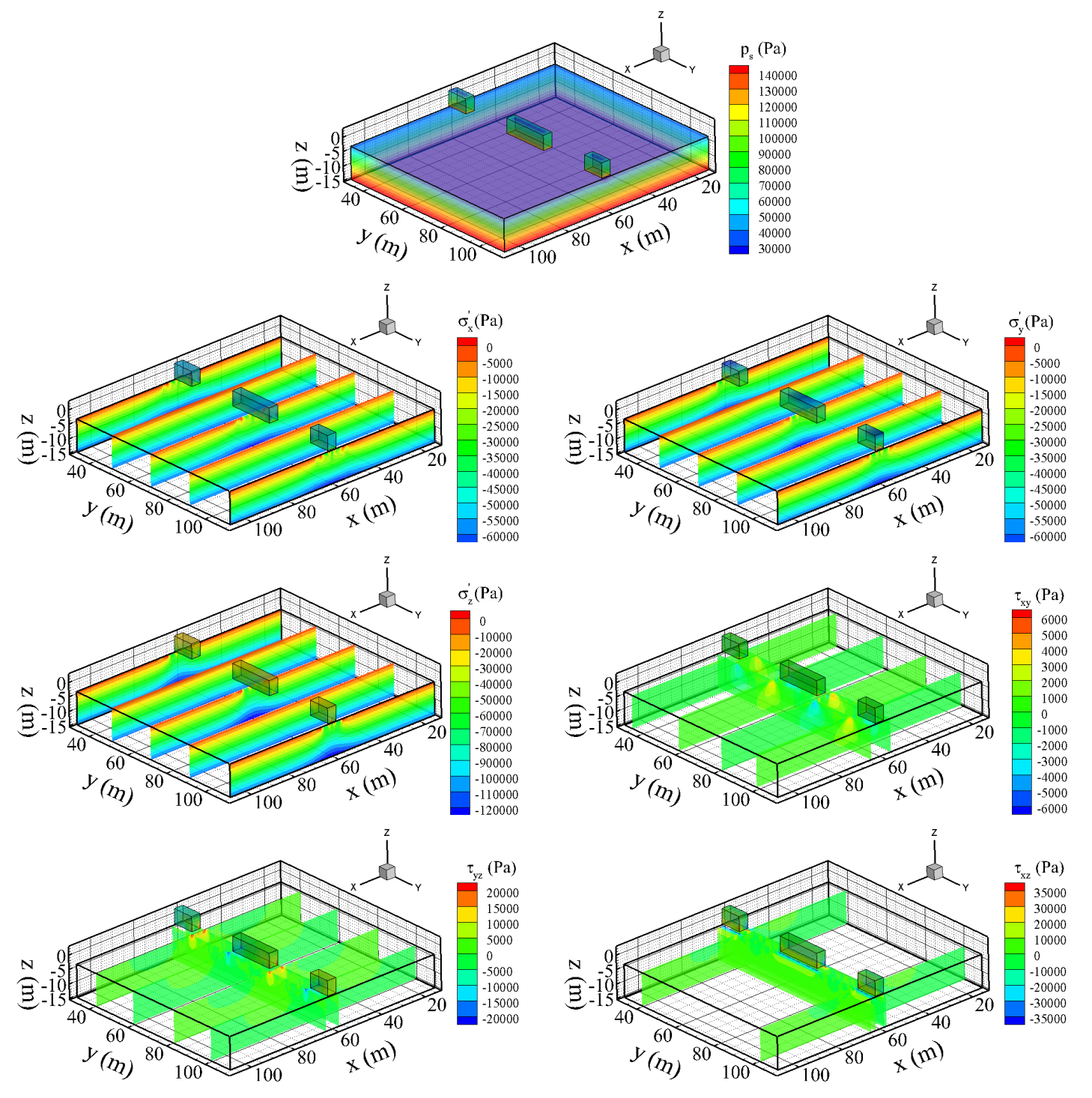

Figure 15: The distribution of pore pressure $\left(p_{s}\right)$, effective stresses $\left(\sigma_{x}^{\prime}, \sigma_{y}^{\prime}\right.$ and $\left.\sigma_{z}^{\prime}\right)$ and shear stresses $\left(\tau_{x y}, \tau_{y z}\right.$ and $\left.\tau_{x z}\right)$ after consolidation process $\left(\mathrm{T}=4.5 \mathrm{~s}, \mathrm{H}=0 \mathrm{~m}, \mathrm{~d}=3.6 \mathrm{~m}, U_{0}=0 \mathrm{~m} / \mathrm{s}\right)$.

until $p^{\prime}$ reaches zero and the seabed foundation liquefies. It indicates that the liquefaction of a elastoplastic seabed foundation does not occur instantaneously, but a progressive process that the soil gradually lose its strength under the cyclic loading. At location A, the seabed foundation can maintain a relatively stable strength in the early stage, then followed by a high rate of decrease in $p^{\prime}$ and $q$ until the seabed liquefies. The stability of seabed foundation decrease uniformly at other locations under the action of waves and currents and the non-linearity is not very obvious. However, they all reach the liquefaction state.

\subsection{Liquefaction around the offshore detached breakwaters}

The liquefaction condition in the seabed foundation is evaluated based on the liquefaction criterion proposed by Jeng and Zhao (2015), which is calculated as follow: 

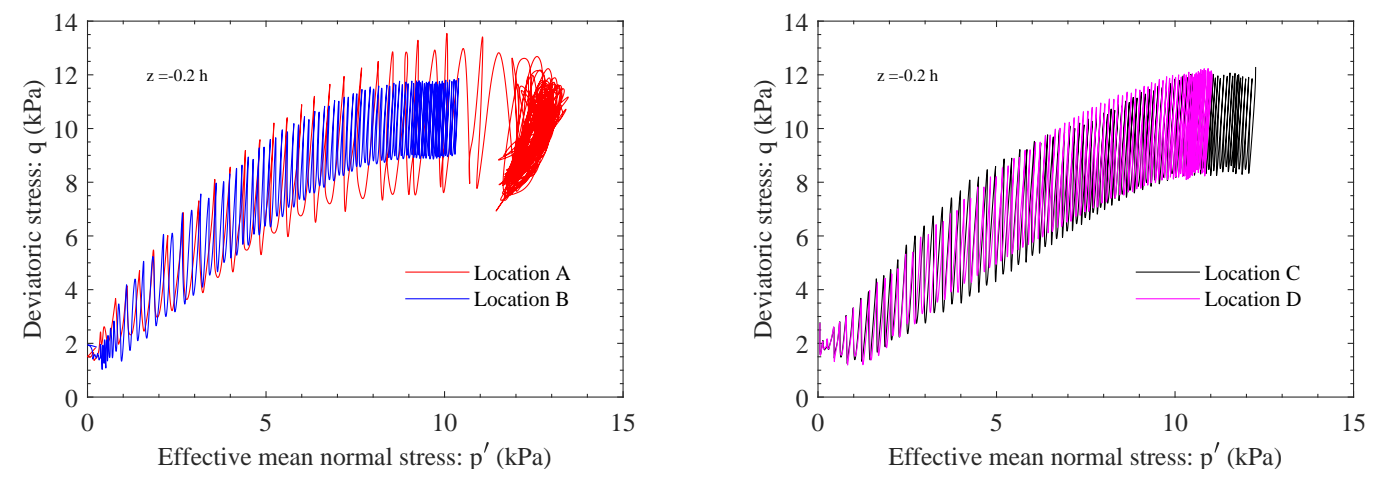

Figure 16: Effective stress paths at locations A, B, C and D subjected to wave and current loading.

$$
\frac{1}{3}\left(\left|\sigma_{x 0}^{\prime}\right|+\left|\sigma_{y 0}^{\prime}\right|+\left|\sigma_{z 0}^{\prime}\right|\right) \leq p_{\text {excess }}
$$

where the left-hand-side denotes the initial mean effective stress and the right-hand-side represents the excess pore pressure caused by the wave loading. Since the residual liquefaction is caused by the weakening in the contact force between the soil particles due to the build-up of the pore pressure, $p_{\text {excess }}$ should be considered as the accumulative value of the pore pressure induced by wave loading.

Figure 17 displays the residual liquefaction zones in the vicinity of breakwaters within a loosely packed poroelastoplastic seabed foundation from $t=250 \mathrm{~s}$ to $t=350 \mathrm{~s}$. Three cross-sections at typical locations are selected: $x$ $=54 \mathrm{~m}$ which is located in front of the breakwaters, $x=60 \mathrm{~m}$ which is just beneath the breakwaters and $x=66 \mathrm{~m}$ which is behind the breakwaters. It is found from the figure that the soil in front of and beneath the breakwaters is most severely liquefied, while the liquefaction of soil behind the breakwaters is relatively less severe due to the protection from the breakwaters, only small areas of liquefaction appeared which concentrate on the gap region between breakwaters. For example, at $t=350 \mathrm{~s}$, the largest liquefaction depth in the slice of $x=54 \mathrm{~m}$ is around $30 \%$ of foundation thickness and less than $20 \%$ in the slice of $x=66 \mathrm{~m}$. It is also found that liquefaction depth beneath the breakwaters is even larger, which brings great threat to the stability of the structure. In terms of time span, the liquefaction zones keep expanding, but the rate of expansion is slowing down.

Figure 18 illustrates the liquefaction depth $\left(L_{\text {depth }}\right)$ within the seabed foundation at $t=300 \mathrm{~s}$. The $3 \mathrm{D}$ effect of wave/current loading around the structures can be clearly observed from the figure. From the figure, the largest $L_{\text {depth }}$ occurs in front of the middle breakwater, which is over $2 \mathrm{~m}$. This is because the strong non-linear interactions between the incident waves, reflected waves and longshore current makes this area more active comparing to other regions. The segmented coast-paralleled breakwaters can partly provide shelter area behind the breakwater, however, due to that the waves can pass through the gaps between the breakwaters and the existence of diffracted 



(a) $x=54 \mathrm{~m}$

(b) $x=60 \mathrm{~m}$

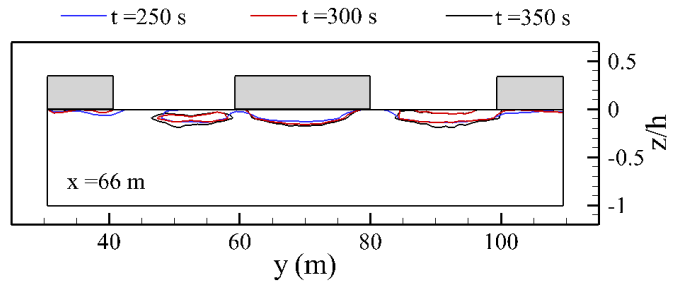

(c) $x=66 \mathrm{~m}$

Figure 17: Liquefaction zones within a loosely packed poro-elastoplastic seabed foundation around breakwaters at $t=250,300$ and $350 \mathrm{~s}$ for three typical slices: (a) $x=54$, (b) $x=60$ and (c) $x=66 \mathrm{~m}$.

waves, there will still be a certain degree of liquefaction behind the breakwaters after a long period of cyclic loading. However, the liquefaction zones behind the breakwaters are relatively shallow and distributed dispersively.

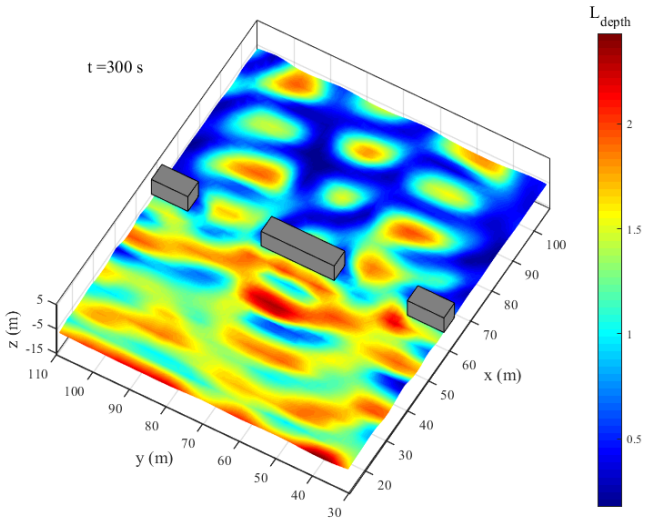

Figure 18: The liquefaction depth within a seabed foundation at $t=300 \mathrm{~s}$.

\subsection{Parametric study}

To quantify the susceptibility of the poro-elastoplastic seabed foundation to liquefaction, a parameter $L_{\text {potential }}$ (liquefaction potential) can be obtained by performing a simple operation on (29):

$$
L_{\text {potential }}=\frac{p_{\text {excess }}}{\frac{1}{3}\left(\left|\sigma_{x 0}^{\prime}\right|+\left|\sigma_{y 0}^{\prime}\right|+\left|\sigma_{z 0}^{\prime}\right|\right)}
$$

Differently, the pore pressure in the poro-elastic foundation is periodic without accumulation, hence, we use parameter $L_{\text {potential }(\max )}$ instead to evaluate the possibility of a poro-elastic seabed to be liquefied. $L_{\text {potential(max) }}$ 
depends on the maximum wave-induced dynamic effective stresses within the whole simulation period, which is calculated as (31) based on the liquefaction criteria proposed by Tsai (1995):

$$
L_{\text {potential }(\max )}=\frac{\frac{1}{3}\left(\sigma_{x d}^{\prime}+\sigma_{y d}^{\prime}+\sigma_{z d}^{\prime}\right)}{\frac{1}{3}\left(\left|\sigma_{x 0}^{\prime}\right|+\left|\sigma_{y 0}^{\prime}\right|+\left|\sigma_{z 0}^{\prime}\right|\right)}
$$

where the numerator represents the mean value of the wave-induced dynamic effective stresses (i.e., the increase of the effective stresses caused by the wave cyclic loading); the denominator represents the initial mean effective stresses.

From the definitions, the higher the $L_{\text {potential }}$, the closer the seabed foundation is to liquefaction. In order to investigate the effects of various parameters on the stability of the seabed foundation around detached breakwaters when subject to waves and longshore currents loading, the liquefaction potential along the depth of the seabed foundation at location A (Zone I) \& B (Zone III) will be discussed for both the poro-elastoplastic and the poroelastic seabed foundation.

\subsubsection{Effect of seabed properties}

Among the soil parameters, the permeability of soil $\left(k_{s}\right)$ and degree of saturation $\left(S_{r}\right)$ are usually the sensitive parameters. $k_{s}$ is an important parameter for measuring the drainage capacity of the soil, which has a great impact on the accumulation rate of the pore water pressure. When soil permeability $\left(k_{s}\right)$ is large, the drainage performance of the soil is good and the pore water pressure build-up rate is low; otherwise, the drainage capacity is bad and the pore water pressure build-up rate is high. The degree of saturation $\left(S_{r}\right)$ is another soil parameter that might affect the seabed foundation response. Although most seabed foundations have degrees of saturation close to fully saturated, the full saturation condition is rare in the real environment, it is common to find air within the seabed soil (Okusa, 1985).

Figure 19 and Figure 20 illustrate the effect of soil permeability $\left(k_{s}\right)$ and degree of saturation $\left(S_{r}\right)$ on the vertical distribution of liquefaction potential $\left(L_{\text {potential }}\right.$ or $\left.L_{\text {potential(max) }}\right)$ at location A and B in the poro-elastoplastic and poro-elastic seabed foundation. The figures show that $k_{s}$ has a more significant impact on the foundation stability compared to $S_{r}$. As been displayed by Figure 19, the liquefaction condition is more severe in the seabed foundation with low $k_{s}$. An interesting finding is that $L_{\text {potential }}$ increases as $k_{s}$ decreases from $10^{-4} \mathrm{~m} / \mathrm{s}$ to $10^{-8}$ $\mathrm{m} / \mathrm{s}$ in the poro-elastoplastic seabed foundation, however, the effect of $k_{s}$ on the foundation stability becomes less sensitive as it reaching $10^{-6} \mathrm{~m} / \mathrm{s}$ and continuing to decrease. This might indicate that the soil liquefaction does not deteriorate indefinitely with the decrease of $k_{s}$. When $k_{s}$ is low to a certain extent, the liquefaction condition reaches its worst case. For the influence of degree of saturation $\left(S_{r}\right)$ in the poro-elastoplastic seabed foundation, there are 
no significant differences between different cases, which indicates that $S_{r}$ has little effect on the stability of poroelastoplastic seabed foundation. Therefore, it can be concluded that in the poro-elastoplastic seabed foundation, the key influence factor among the soil properties on the foundation stability is the soil permeability $\left(k_{s}\right)$.

To see the difference between poro-elastic model and poro-elastoplastic model, we also consider the poroelastic seabed, which only have oscillatory liquefaction rather than residual liquefaction. It is observed that the liquefaction potential in the poro-elastic seabed foundation is much smaller than that in the poro-elastoplastic seabed foundation under the same wave/current loading, the value of $L_{\text {potential }}$ of the former foundation is only about $10 \%$ of the latter foundation. For example, in the case of seabed with $k_{s}=10^{-4} \mathrm{~m} / \mathrm{s}, L_{\text {potential }}$ max is less than 0.25 , indicating that the poro-elastic seabed foundation is not liquefied under such condition. Different from that in poro-elastoplastic seabed foundation, $S_{r}$ has quite an impact on the stability of poro-elastic seabed foundation.

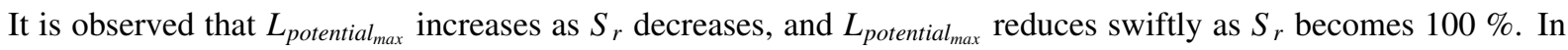
summary, compared to the poro-elastoplastic seabed foundation, the liquefaction has minor effects on the structure stability in a poro-elastic seabed foundation. However, the liquefaction may still deteriorate the scouring around breakwaters and affect the stability of coastal structures. Comparing among the different locations, location A has a deeper liquefaction depth $\left(L_{\text {depth }}\right)$ than location B. For example, in the case of seabed foundation with $k_{s}=10^{-4}$ $\mathrm{m} / \mathrm{s}, L_{\text {depth }}$ at location A reaches over $20 \%$ of foundation thickness. Due to the protection from the middle breakwater, location B only has a $L_{\text {depth }}$ of $5 \%$ of foundation thickness. It is also found from the figures that the liquefaction potential is smallest at seabed bottom $\left(L_{\text {potential }}=0\right)$ and gradually increases along the depth to the seabed surface, in other words, the closer to the seabed surface, the greater the liquefaction potential value.

Two sets of PZIII constitutive model parameters, representing the loose deposited poro-elastoplastic seabed foundation with relative density $\left(D_{r}\right)$ equals to $40 \%$ and the dense deposited poro-elastoplastic seabed foundation with $D_{r}$ equals to $60 \%$, are used to study the foundation stability around the breakwaters. Figure 21 shows the vertical distribution of $L_{\text {potential }}$ in these two types of seabed foundation at $t=350 \mathrm{~s}$. It can be seen from the figure that $L_{\text {depth }}$ in loose sand is almost twice as deep as in dense sand at each location. For instance, $L_{\text {depth }}$ at location behind the middle breakwater (i.e., location B $(x=65 \mathrm{~m}, y=70 \mathrm{~m})$ ) reaches nearly $10 \%$ and $20 \%$ of the seabed thickness in dense foundation and loose seabed foundation, respectively. This suggests that there is a higher chance to severe liquefaction in loose seabed foundation with small $D_{r}$ because loose sand is easier to be contracted under cyclic loading and causes greater pore pressure accumulation. 

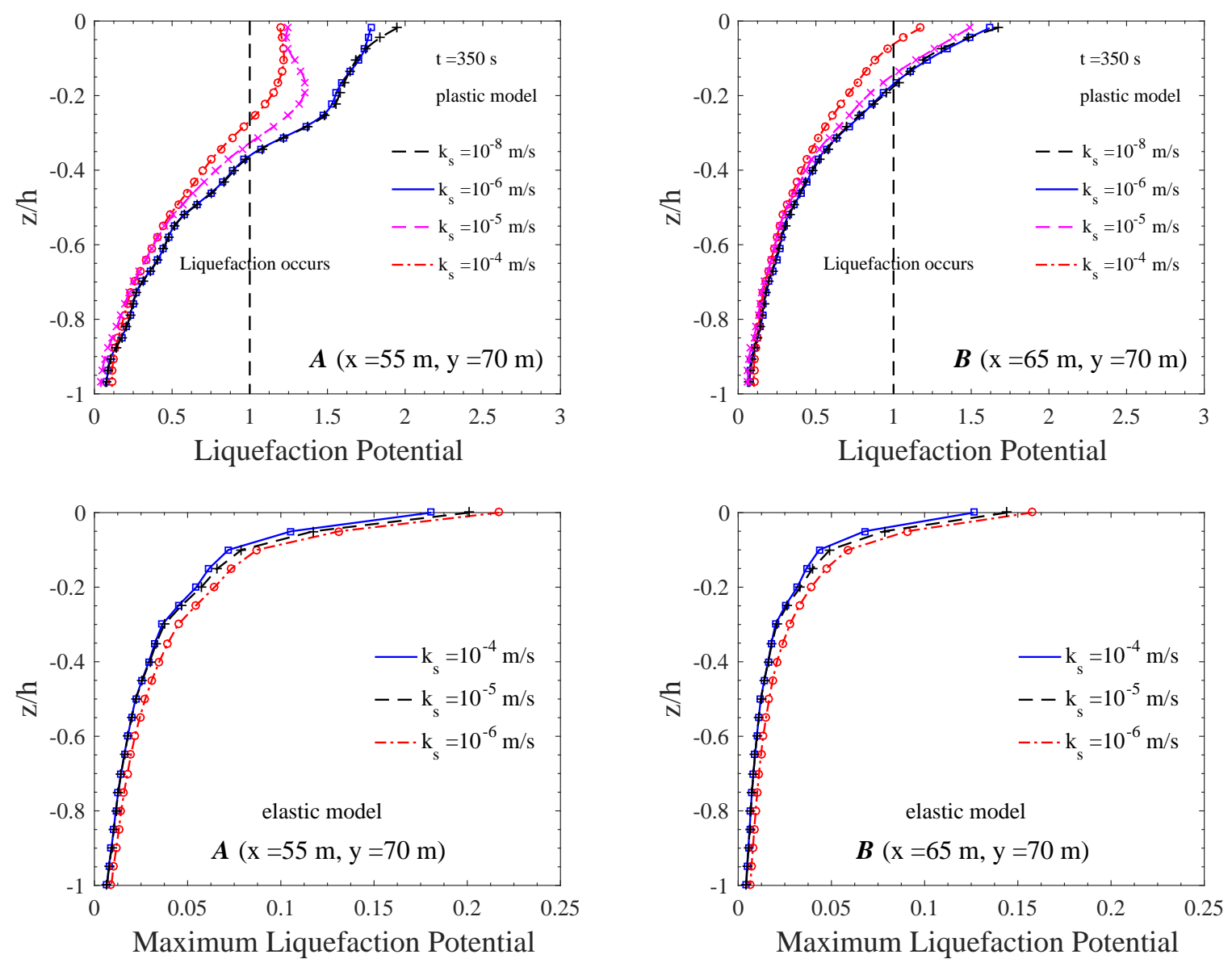

Figure 19: Vertical distribution of the liquefaction potential for various soil permeability $\left(k_{s}=10^{-4} \mathrm{~m} / \mathrm{s}, k_{s}=10^{-5} \mathrm{~m} / \mathrm{s}, k_{s}=10^{-6} \mathrm{~m} / \mathrm{s}\right.$ and $\left.k_{s}=10^{-8} \mathrm{~m} / \mathrm{s}\right)$ at location $\mathrm{A}(x=55 \mathrm{~m}, y=70 \mathrm{~m})$ and B $(x=65 \mathrm{~m}, y=70 \mathrm{~m})$ within the poro-elastoplastic and poro-elastic seabed foundation $\left(\mathrm{T}=4.5 \mathrm{~s}, \mathrm{H}=2.4 \mathrm{~m}, \mathrm{~d}=3.6 \mathrm{~m}, U_{0}=0.6 \mathrm{~m} / \mathrm{s}\right)$.

\subsubsection{Effect of wave characteristics}

Figure 22 represents the vertical distribution of $L_{\text {potential }}$ and $L_{\text {potential } m_{\max }}$ for various combination of waves and longshore currents $\left(U_{0}=-0.6 \mathrm{~m} / \mathrm{s}, U_{0}=0 \mathrm{~m} / \mathrm{s} \& U_{0}=-0.3 \mathrm{~m} / \mathrm{s}\right)$ in poro-elastoplastic seabed foundation and poroelastic seabed foundation, respectively. As illustrated in the figures, the presence of the perpendicular longshore currents increase the liquefaction potential along the depth of seabed foundation and it becomes larger as the magnitude of velocity becomes larger. The influence is mainly reflected in the shallow soil layer near the seabed surface, while the influence on the deep soil is relatively small, especially in the poro-elastoplastic seabed foundation. Therefore, it can be concluded that the effect of wave-current interaction is more significant in the upper seabed layer with stronger longshore currents and the current has little effect on the deeper seabed stability.

Among the wave parameters, in addition to the ocean currents, wave height $(H)$ and wave period $(T)$ can also affect the stability of the foundation. As illustrated in Figures 23, the vertical distribution of liquefaction potential 

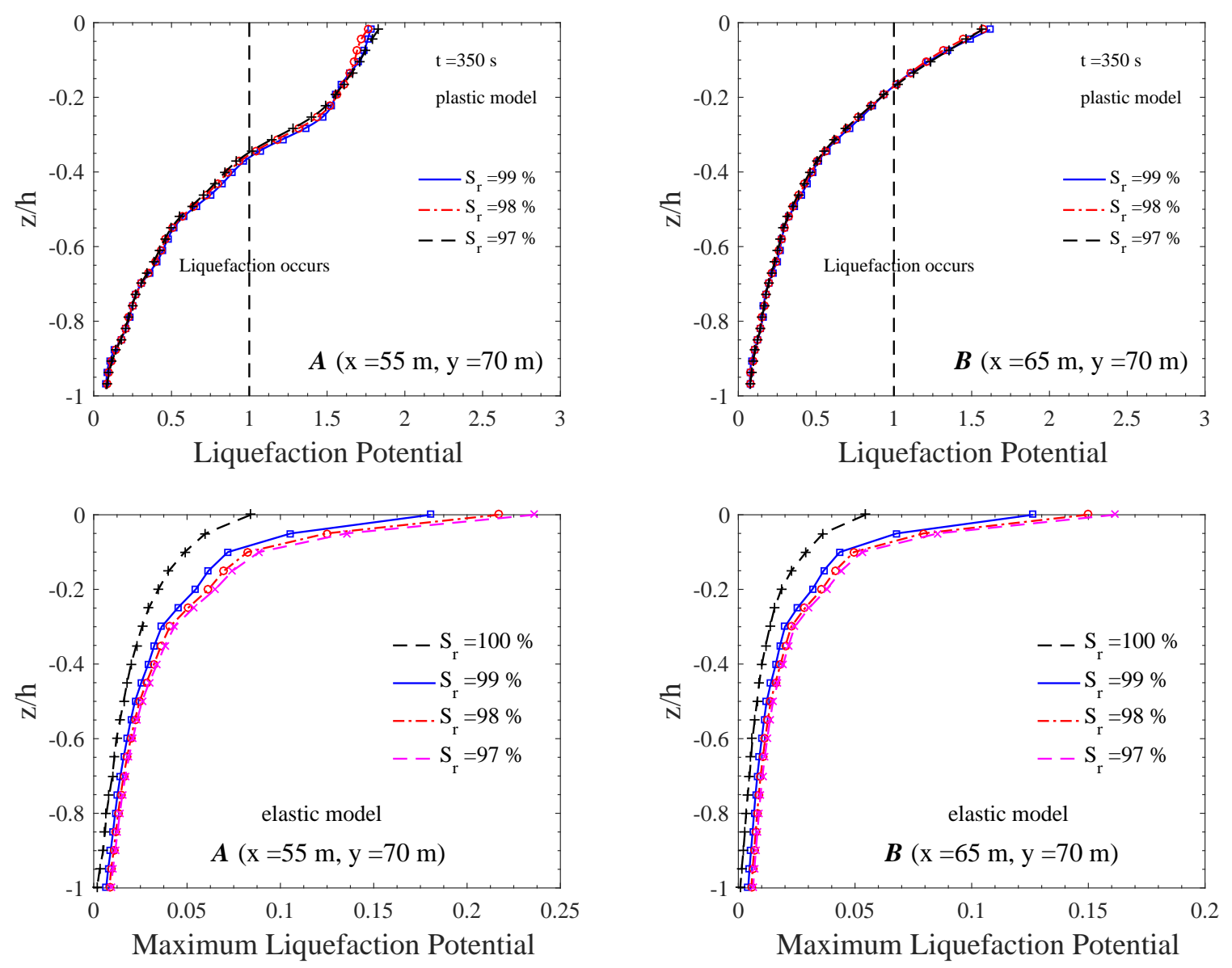

Figure 20: Vertical distribution of the liquefaction potential for various degree of saturation $\left(S_{r}=99 \%, S_{r}=98 \%\right.$ and $\left.S_{r}=97 \%\right)$ at location A $(x=55 \mathrm{~m}, y=70 \mathrm{~m})$ and $\mathrm{B}(x=65 \mathrm{~m}, y=70 \mathrm{~m})$ within the poro-elastoplastic and poro-elastic seabed foundation $(\mathrm{T}=4.5 \mathrm{~s}, \mathrm{H}=2.4 \mathrm{~m}, \mathrm{~d}=3.6$ $\left.\mathrm{m}, U_{0}=0.6 \mathrm{~m} / \mathrm{s}\right)$.


Figure 21: Vertical distribution of the liquefaction potential ( $\left.L_{\text {potential }}\right)$ at location A $(x=55 \mathrm{~m}, y=70 \mathrm{~m})$ and B $(x=65 \mathrm{~m}, y=70 \mathrm{~m})$ within the loosely deposited poro-elastoplastic seabed foundation and densely deposited poro-elastoplastic seabed foundation at $t=350 \mathrm{~s}(\mathrm{~T}=4.5 \mathrm{~s}, \mathrm{H}$ $\left.=2.4 \mathrm{~m}, \mathrm{~d}=3.6 \mathrm{~m}, U_{0}=0.6 \mathrm{~m} / \mathrm{s}\right)$. 

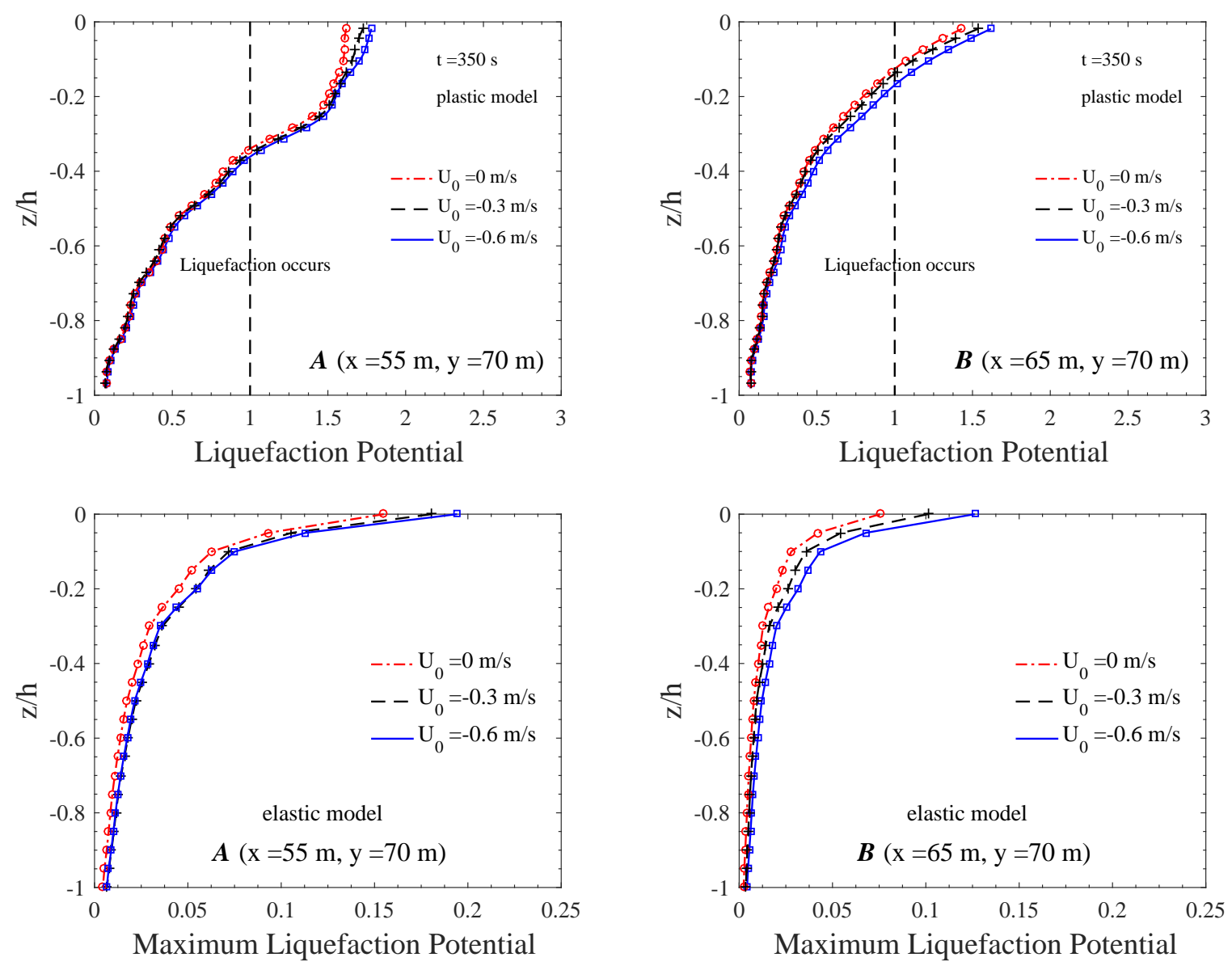

Figure 22: Vertical distribution of the liquefaction potential for various longshore currents conditions $\left(U_{0}=-0.6 \mathrm{~m} / \mathrm{s}, U_{0}=0 \mathrm{~m} / \mathrm{s}\right.$ and $U_{0}=-0.3$ $\mathrm{m} / \mathrm{s})$ at location A $(x=55 \mathrm{~m}, y=70 \mathrm{~m})$ and B $(x=65 \mathrm{~m}, y=70 \mathrm{~m})$ within the poro-elastoplastic and poro-elastic seabed foundation $(\mathrm{T}=4.5 \mathrm{~s}$, $\mathrm{H}=2.4 \mathrm{~m}, \mathrm{~d}=3.6 \mathrm{~m})$.

in the poro-elastoplastic and poro-elastic seabed foundation increases as the increase of $H$ and $T$. It implies that waves with larger height and longer period can pose more threats on foundation stability around the structures than a smaller height and shorter period wave under the same soil condition. As an example, at location A which is in front of the middle breakwater, the liquefaction depth $\left(L_{\text {depth }}\right)$ is about $25 \%$ of foundation thickness for $H=1.7$ m, and reaches $35 \%$ of foundation thickness for $H=2.4 \mathrm{~m}$; and the difference of $L_{\text {depth }}$ between the case with $T$ $=3.5 \mathrm{~s}$ and $T=4.5$ can reach up to $20 \%$. This may be explained by the fact that the wave steepness $(H / L)$ becomes larger for a larger $H$ and the relative water depth $(d / L)$ becomes smaller for a longer $T$ in the same water depth.

\section{Conclusions}

This study numerically investigates the dynamic behaviour and the liquefaction potential of the seabed foundation in the vicinity of the offshore detached breakwaters under combined waves and perpendicular longshore currents. The present study adds the following new contributions to the field: 

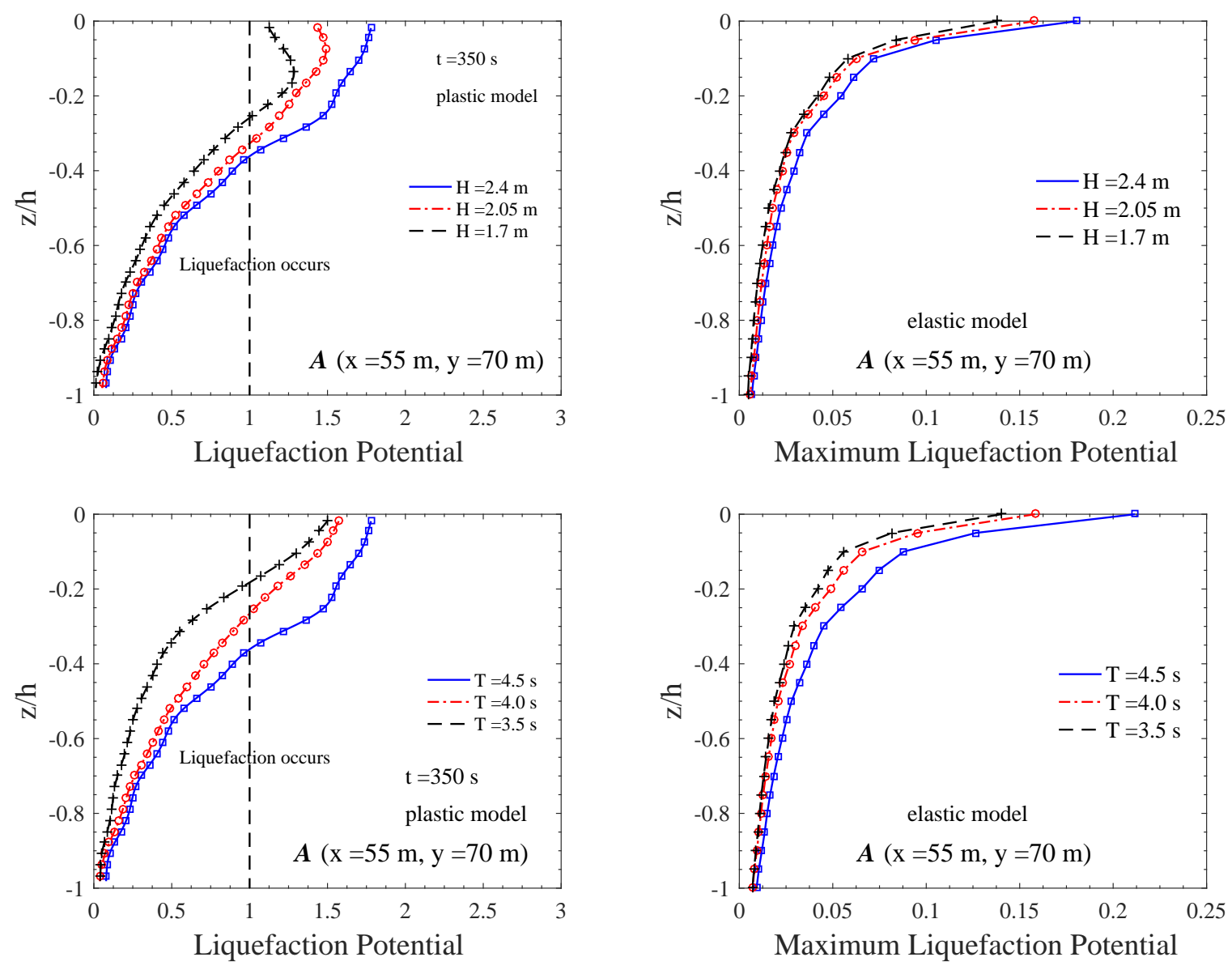

Figure 23: Vertical distribution of the liquefaction potential for various wave height $(H=2.4 \mathrm{~m}, H=2.05 \mathrm{~m}$ and $H=1.7 \mathrm{~m})$ and wave period $(T$ $=4.5 \mathrm{~s}, T=4.0 \mathrm{~s}$ and $T=3.5 \mathrm{~s})$ at location A $(x=55 \mathrm{~m}, y=70 \mathrm{~m})$ within the poro-elastoplastic and poro-elastic seabed foundation $(\mathrm{d}=3.6 \mathrm{~m}$, $\left.U_{0}=0.6 \mathrm{~m} / \mathrm{s}\right)$.

- From 2D to 3D: when studying the FSSI around breakwaters, many scholars simplified their simulation object as a typical truck section due to the limitations of the 2D models. The $3 \mathrm{D}$ interactions like wave diffraction, waves \& currents at different angles of incidence from structures are generally ignored. As a complement to the existing 3D cases, this study investigates the three-dimensional hydrodynamics and seabed foundation responses around the segmented offshore detached breakwaters using a 3D numerical model.

- Loose seabed foundation with plastic soil behaviour: Loose soil usually produces plastic dynamic behaviours such as nonlinear deformation and soil skeleton contraction under cyclic wave loading due to its poor soil mechanical properties, therefore, it is not suitable to be used as a foundation for offshore structures. However, because of its wide distribution, engineers have no other choice when it comes to the location of the structure. Therefore, it is necessary to understand the dynamic response and liquefaction of loose soil 
foundation in Marine environment. Majority of the existing studies of FSSI around breakwaters focused on the elastic behaviours in the seabed foundation. The present study adopts a poro-elastoplastic constitutive model to describe the degradation of stiffness and liquefaction potential of a loose seabed foundation around breakwaters.

- Combined wave and longshore current loading: Many researches for FSSI around breakwaters used wave loading without considering currents, but different forms of currents exit in the real environment and constantly alter the wave conditions in the vicinity of structures and further exacerbate the instability of seabed foundation. This study includes longshore currents that are parallel to the shoreline but perpendicular to the waves propagation and examine their effects on the surrounding hydrodynamic properties and liquefaction potential in the seabed foundation.

Using different constitutive models, the stability of both poro-elastic seabed foundation and poro-elastoplastic seabed foundation was examined for various wave/soil parameters. Based on the numerical results, the main conclusions can be drawn as follow:

1. The 3D fluid motion around the offshore detached breakwaters is successfully modelled. The non-linear interactions among the waves, longshore currents and the structures are observed in this study. The presence of the longshore currents has a significant impact on the hydrodynamic process, which they exacerbate the wave motions, increase the irregularity and constantly alter the wave propagation.

2. The stress status of porous seabed foundation after the consolidation process is determined first and applied as the initial condition in the dynamic analysis of FSSI problem. It is found that the construction of breakwaters can significantly increase the effective stress and shear stress in the nearby area. Ignoring the consolidation process would overestimate the liquefaction potential within the seabed foundation.

3. The significant drops of stress paths indicate that the loose seabed foundation around the offshore detached breakwaters experienced a degradation of stiffness under successive loading cycles as the risk of structure failure is increasing. After a number of loading cycles, the most severe liquefaction occurred on the seaward side of the breakwaters (Zone I), and the mildest on the shoreward side (Zone III) due to energy depletion caused by the breakwaters. 
4. Numerical results show that, under the same wave/current loading, the liquefaction potential within the poroelastoplastic seabed foundation is much larger than that in the poro-elastic seabed foundation, structures built on the former type of foundation have a greater likelihood of collapse. Therefore, it is suggested that engineers should pay more attention to this kind of soil during the design and construction of breakwater and take reinforcement measures when necessary.

5. Parametric studies show that the soil permeability $k_{s}$ has a significant influence on the development of liquefaction in the seabed foundation. The liquefaction condition is more severe in the seabed foundation with low $k_{s}$ and the effect of $k_{s}$ on the foundation stability becomes less sensitive as it is lower than $10^{-6} \mathrm{~m} / \mathrm{s}$. Additionally, the longshore currents tend to exacerbate the wave field and increase the risk of liquefaction of seabed foundation, especially the shallow soil layer near the surface. The relative density of soil, wave height and wave period also have a remarkable impact on the liquefaction potential in the seabed foundation.

\section{Credit authorship contribution statement}

Lin Cui: Methodology, Data curation, Visualization, investigation, validation, writing-original draft; DongSheng Jeng: Conceptuation, Methodology, Writing-review \& editing, supervision; Junwie Liu: Visualization, Writing-review \& editing.

\section{Declaration of competing interest}

The authors declare that they have no known competing financial interests or personal relationships that could have appeared to influence the work reported in this paper.

\section{References}

Chang, S.C., Lin, J.G., Chien, L.K., Chiu, Y.F., 2007. An experimental study on non-linear progressive waveinduced dynamic stresses in seabed. Ocean Engineering 34, 2311-2329.

Fredsøe, J., Sumer, B.M., 1997. Scour at the round head of a rubble-mound breakwater. Coastal engineering 29, $231-262$.

Higuera, P., 2015. Application of computational fluid dynamics to wave action on structures. Ph.D. thesis. University of Cantabria.

Higuera, P., Lara, J.L., Losada, I.J., 2013. Realistic wave generation and active wave absorption for Navier-Stokes models: Application to OpenFOAM. Coastal Engineering 71, 102-118. 
Jeng, D.-S., 1996. Wave-induced liquefaction potential at the tip of a breakwater: an analytical solution. Applied Ocean Research 18, 229-241. doi:10.1016/S0141-1187(96)00033-8.

Jeng, D.-S., Cha, D., Lin, Y., Hu, P., 2000. Analysis on pore pressure in an anisotropic seabed in the vicinity of a caisson. Applied Ocean Research 22, 317-329. doi:10.1016/S0141-1187(00)00023-7.

Jeng, D.-S., 2018. Mechanics of wave-seabed-structure interactions: modelling, processes and applications. Cambridge University Press. doi:10.1017/9781316672266.

Jeng, D.-S., Cha, D., Lin, Y., Hu, P., 2001. Wave-induced pore pressure around a composite breakwater. Ocean Engineering 28, 1413-1435. doi:10.1016/S0029-8018(00)00059-7.

Jeng, D.-S., Ou, J., 2010. 3D models for wave-induced pore pressures near breakwater heads. Acta Mechanica 215, 85-104. doi:10.1007/200707-010-0303-z.

Jeng, D.-S., Schacht, C., Lemckert, C., 2005. Experimental study on ocean waves propagating over a submerged breakwater in front of a vertical seawall. Ocean Engineering 32, 2231-2240. doi:10.1016/j.oceaneng.2004.12.015.

Jeng, D.-S., Seymour, B., 2007. Simplified analytical approximation for pore-water pressure buildup in marine sediments. Journal of Waterway, Port, Coastal, and Ocean Engineering, ASCE 133, 309-312. doi:10.1061/(ASCE)0733-950X(2007)133:4(309).

Jeng, D.-S., Zhao, H., 2015. Two-dimensional model for accumulation of pore pressure in marine sediments. Journal of Waterway, Port, Coastal, and Ocean Engineering, ASCE 141, 04014042.

Kudella, M., Oumeraci, H., de Groot, M., Meijers, P., 2006. Large-scale experiments on pore pressure generation underneath a caisson breakwater. Journal of Waterways, Port, Coastal and Ocean Engineering, ASCE 132, $310-324$.

Kumagai, T., Foda, M.A., 2002. Analytical model for response of seabed beneath composite breakwater to wave. Journal of Waterway, Port,APOR-D-21-00039 Coastal, and Ocean Engineering, ASCE 128, 62-71.

Li, J., Jeng, D.-S., 2008. Response of a porous seabed around breakwater heads. Ocean Engineering 35, 864-886. doi:10.1016/j.oceaneng.2008.01.021.

Liao, C., Tong, D., Jeng, D.-S., Zhao, H., 2018. Numerical study for wave-induced oscillatory pore pressures and liquefaction around impermeable slope breakwater heads. Ocean Engineering 157, 364-375. 
Mizutani, N., Mostafa, A.M., Iwata, K., 1998. Nonlinear regular wave, submerged breakwater and seabed dynamic interaction. Coastal Engineering 33, 177-202.

Mynett, A.E., Mei, C.C., 1982. Wave-induced stresses in a saturated poroelastic seabed beneath a rectangular caisson. Géotechnique 3APOR-D-21-00039 2, 235-248.

Okusa, S., 1985. Wave-induced stress in unsaturated submarine sediments. Géotechnique 35, 517-532.

Oumeraci, H., 1994. Review and analysis of vertical breakwater failures-lessons learned. Coastal Engineering 22, 3-29.

Pastor, M., Zienkiewicz, O.C., Chan, A.H.C., 1990. Generalized plasticity and the modeling of soil behaviour. International Journal for Numerical and Analytical Methods in Geomechanics 14, 151-190.

Qi, W.G., Li, C.F., Jeng, D.-S., Gao, F.P., Liang, Z., 2019. Combined wave-current induced excess pore-pressure in a sandy seabed: APOR-D-21-00039 Flume observations and comparisons with theoretical models. Coastal Engineering 147, 89-98.

Sassa, S., Sekiguchi, H., 1999. Wave-induced liquefaction of beds of sand in a centrifuge. Géotechnique 49, $621-638$.

Seed, H.B., Rahman, M.S., 1978. Wave-induced pore pressure in relation to ocean floor stability of cohesionless soils. Marine Geotechnology 3, 123-150.

Stoker, J.J., 1957. Water waves. Interscience Publishers, Inc., New York.

Sumer, B.M., 2014. Liquefaction around Marine Structures. World Scientific, New Jersey.

Tsai, C.P., 1995. Wave-induced liquefaction potential in a porous seabed in front of a breakwater. Ocean Engineering $22,1-18$.

Tsai, Y.T., McDougal, W.G., Sollitt, C.K., 1990. Response of finite depth seabed to waves and caisson motion. Journal of Waterways, Port and Coastal and Ocean Engineering, ASCE 116, 1-20.

Ulker, M., Rahman, M.S., Guddati, M.N., 2010. Wave-induced dynamic response and instability of seabed around caisson breakwater. Ocean Engineering 37, 1522-1545. 
Ulker, M.B.C., Rahman, M.S., Guddati, M.N., 2012. Breaking wave-induced response and instability of seabed around caisson breakwater. International Journal for Numerical and Analytical Methods in Geomechanics 36, 362-390.

Van Gent, M., 1995. Porous flow through rubble-mound material. Journal of Waterway, Port, Coastal, and Ocean Engineering, ASCE 121, 176-181.

Yamamoto, T., Koning, H., Sellmeijer, H., Hijum, E.V., 1978. On the response of a poro-elastic bed to water waves. Journal of Fluid Mechanics 87, 193-206.

Ye, J., Jeng, D.-S., Wang, R., Zhu, C., 2015. Numerical simulation of the wave-induced dynamic response of poroelastoplastic seabed foundations and a composite breakwater. Applied Mathematical Modelling 39, 322-347.

Ye, J., Jeng, D.-S., Chan, A.H.C., Wang, R., Zhu, Q.C., 2016. 3d integrated numerical model for fluid-structuresseabed interaction (FSSI): Elastic dense seabed foundation. Ocean Engineering 115, 107-122.

Ye, J., Jeng, D.-S., Liu, P.L.F., Chan, A.H.C., Wang, R., Zhu, C., 2014. Breaking wave-induced response of composite breakwater and liquefaction in seabed foundation. Coastal Engineering 85, 72-86.

Zhao, H., Jeng, D.-S., Zhang, J., Liao, C., Zhang, H., Zhu, J., 2017. Numerical study on loosely deposited foundation behavior around a composite breakwater subject to ocean wave impact. Engineering Geology 227, $121-138$.

Zhao, H., Jeng, D.-S., Zhang, Y., Zhang, J., Zhang, H., Zhang, C., 2013. 3d numerical model for wave-induced seabed response around breakwater heads. Geomechanics and Engineering 5, 595-611.

Zhao, H., Liang, Z., Jeng, D.-S., Zhu, J., Guo, Z., Chen, W., 2018. Numerical investigation of dynamic soil response around a submerged rubble mound breakwater. Ocean Engineering 156, 406-423.

Zhao, H.Y., Jeng, D.-S., 2015. Numerical study of wave-induced soil response in a sloping seabed in the vicinity of a breakwater. Applied Ocean Research 51, 204-221.

Zhao, H.Y., Zhu, J.F., Liu, X.L., Jeng, D.-S., Zheng, J.H., Zhang, J.S., 2020a. Numerical investigation of dynamic soil response around a submerged rubble mound breakwater: Ii. loose sandy seabed. Ocean Engineering 215, 107891. doi:https://doi.org/10.1016/j.oceaneng.2020.107891. 
574

Zhao, H.Y., Zhu, J.F., Zheng, J.H., Zhang, J.S., 2020b. Numerical modelling of the fluid-seabed-structure interactions considering the impact of principal stress axes rotations. Soil Dynamics and Earthquake Engineering 136, 106242. doi:https://doi.org/10.1016/j.soildyn.2020.106242.

Zienkiewicz, O.C., Chang, C.T., Bettess, P., 1980. Drained, undrained, consolidating and dynamic behaviour assumptions in soils. Géotechnique 30, 385-395. 